\title{
EchoGéo
}

$54 \mid 2020$

Varia

\section{Cartographier par télédétection l'occupation du sol et ses changements}

Application à l'analyse de la dynamique des paysages forestiers sénégambiens entre 1972 et 2016

Ibrahima Diédhiou, Catherine Mering, Oumar Sy et Tidiane Sané

\section{CpenEdition}

Journals

Édition électronique

URL : https://journals.openedition.org/echogeo/20510

DOI : $10.4000 /$ echogeo.20510

ISSN : 1963-1197

Éditeur

Pôle de recherche pour l'organisation et la diffusion de l'information géographique (CNRS UMR 8586)

Référence électronique

Ibrahima Diédhiou, Catherine Mering, Oumar Sy et Tidiane Sané, «Cartographier par télédétection l'occupation du sol et ses changements », EchoGéo [En ligne], 54 | 2020, mis en ligne le 31 décembre 2020, consulté le 21 septembre 2021. URL : http://journals.openedition.org/echogeo/20510 ; DOI : https://doi.org/10.4000/echogeo.20510

Ce document a été généré automatiquement le 21 septembre 2021.

EchoGéo est mis à disposition selon les termes de la licence Creative Commons Attribution - Pas d'Utilisation Commerciale - Pas de Modification 4.0 International (CC BY-NC-ND) 


\section{Cartographier par télédétection l'occupation du sol et ses changements}

Application à l'analyse de la dynamique des paysages forestiers

sénégambiens entre 1972 et 2016

Ibrahima Diédhiou, Catherine Mering, Oumar Sy et Tidiane Sané

\section{Introduction}

1 La possibilité offerte par la télédétection spatiale d'observer de façon continue la surface de la Terre a permis de créer de grandes bases de données dont l'exploitation fournit des informations sur l'état des ressources naturelles, des écosystèmes locaux et de leur évolution. Dans les régions soudaniennes soumises à des changements socioenvironnementaux rapides (Alexandre et Mering 2019; Fayama et al., 2020), le suivi de l'occupation du sol est capital pour établir un diagnostic et mieux comprendre les causes de la modification du couvert végétal et ses conséquences. À ce titre, les cartes d'occupation et de changements du sol constituent de véritables outils de planification et d'aide à la décision surtout en matière de gestion et de préservation des ressources naturelles et des écosystèmes. Or, pour obtenir ces cartes, plusieurs approches méthodologiques capables d'exploiter au mieux les images satellites existent. Parmi celles-ci, l'analyse multi-temporelle de l'occupation du sol, considérée comme l'une des plus utilisées (Cohen et Fiorella., 1998 ; Lupo et al.,2001 ; Petit et Lambin., 2001 ; Hayes et Sader, 2001 ; Coppin et al., 2004 ; Lu et al., 2004 ; Deng et al., 2008; Fichera et al., 2012 ; Hernandez et Shi., 2018; Si Salah et al., 2020), réside dans sa capacité à prendre en compte la répartition spatiale des changements (Franklin et al., 2002; Griffith et al., 2003 ; Kpedenou et al., 2017). L'objectif du travail présenté ici étant de cartographier les changements majeurs des paysages forestiers au cours d'une période couvrant presque un demi-siècle, nous nous sommes largement inspirés des expériences précédentes pour exploiter les séries temporelles du programme Landsat dont l'accès aux images 
d'archive offre la possibilité d'une telle rétrospective. L'intérêt d'une telle étude est de caractériser l'état des formations végétales et leurs dynamiques spatio-temporelles afin de mieux identifier les menaces qui pèsent sur les ressources naturelles qu'il s'agisse de la péjoration climatique ou de la pression anthropique causées par la demande accrue en bois d'énergie et en produits ligneux, ou encore par l'intensification des pratiques agricoles ou l'urbanisation (Binzangi et al.,1994; Ozer., 2004; Ariori, Ozer, 2005 ; Montagne, 2016 ; Sikuzani et al., 2017 ; Peltier., 2019, Bindu, 2019).

2 La plupart des études sur la dynamique d'évolution des paysages de cette partie des Rivières du Sud (Cormier Salem, 1999) qu'est la Sénégambie méridionale, mettent l'accent sur l'échelle locale pour effectuer le suivi de la mangrove (Tendeng et al., 2016; Soumaré et al., 2020,) soit des formations boisées de terres ferme (Solly et al., 2018) à travers une approche supervisée ou non supervisée. Les travaux qui ont concerné à la fois les deux types de milieu aux échelles infra (Solly et al., 2020) ou supra-régionales (Andrieu et Mering, 2008) privilégient en général l'approche non supervisée. L'étude présentée ici se situe dans la continuité de cette dernière approche éclairée toutefois par le recours à des réalités terrain datant d'une période récente. La cartographie des changements qui en est issue vise à répondre aux questions portant sur la dynamique d'évolution des paysages forestiers sénégambiens entre 1972 et 2016. Chacune des étapes de la démarche allant de l'analyse des images satellite à la cartographie des changements a été détaillée de façon à pouvoir être évaluée voire reproduite.

\section{Présentation de la région d'étude}

3 La Sénégambie méridionale est une région transfrontalière d'environ $20753,27 \mathrm{~km}^{2}$ située entre les bassins des fleuves Casamance (Sénégal) et de la Gambie maritime (illustration 1). Le choix d'une telle zone d'étude à cheval entre le Sénégal et la Gambie se justifie ici par la volonté de pouvoir appréhender les éventuelles répercussions sur et l'évolution des paysages forestiers des politiques publiques en matière de gestion environnementale et de développement rural qui ont été très différentes d'un pays à l'autre durant la période étudiée (Diedhiou, 2019). Une autre particularité de cette région transfrontalière est que, durant la période étudiée, elle a été le théâtre d'un conflit armé entre l'État sénégalais et le Mouvement des Forces Démocratiques de la Casamance $(\mathrm{MFDC})^{1}$ qui a eu un impact important sur l'économie locale et l'environnement (Marut, 2011 ; Sène, 2019).

4 La région se caractérise, d'un point de vue climatique, selon la saison, par l'alternance, entre la mousson et l'alizé maritime sur le littoral, et l'harmattan sur le continent. Sa position de Finistère ouest-africain combinée à cette alternance de flux, confère à la Sénégambie méridionale ce climat singulier qui se caractérise par d'importantes quantités de pluies (entre 1000 et $1400 \mathrm{~mm}$ par an en moyenne) durant la saison pluvieuse (Soumare et al., 2020). 
Illustration 1 - Localisation de la zone d'étude

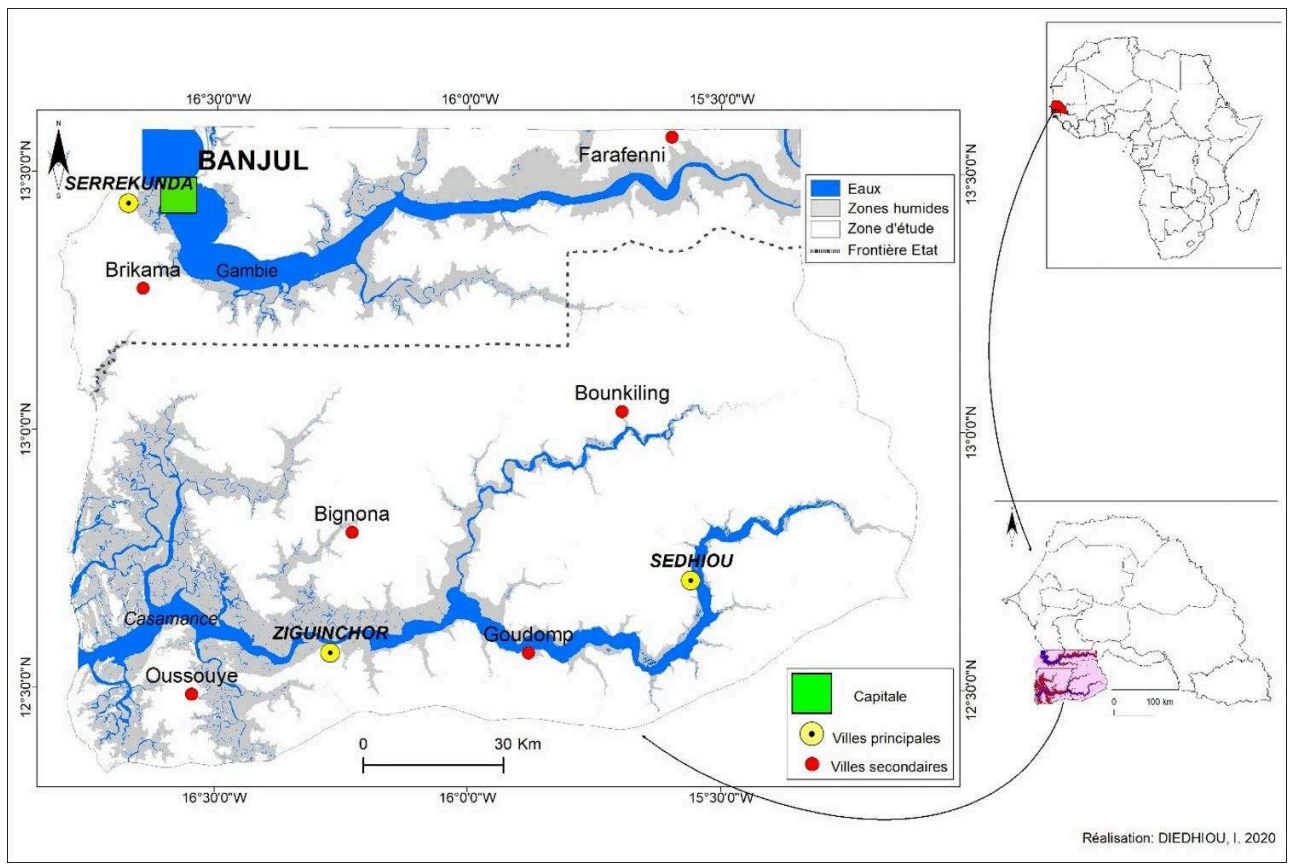

5 De telles conditions climatiques ont favorisé le développement d'un couvert végétal luxuriant ${ }^{2}$ qui a longtemps fait la renommée de cette région et qui a favorisé l'essor de l'agriculture pluviale. Outre l'importance de la pluviométrie, ce territoire est parcouru par un réseau hydrographique dense composé de deux grands cours d'eau, la Casamance et la Gambie, ainsi que d'innombrables affluents, marigots et bolons ${ }^{3}$. Comme dans le reste des Rivières du sud, deux catégories de milieux physiques peuvent être distingués au sein de cette région : un milieu humide de marais maritimes et de vasières le long du littoral et des cours d'eau abritant massifs de mangroves, tannes ${ }^{4}$ et rizières inondées et un milieu de terre ferme où se pratique une agriculture pluviale variée au sein de formations boisées de densité variable entre forêt galerie à la savane ouverte.

\section{Les données de télédétection}

Les données utilisées dans cet article proviennent des différents capteurs Landsat comme Multi Spectral Scanner (MSS), Thematic Mapper (TM), Enhanced Thématic Mapper (ETM+), et Operational Land Imager (OLI). Ces séries d'images ont été prises pour l'essentiel à une période de l'année où la probabilité d'acquérir des images nettes et contrastées est la plus élevée (Demaze, 2007). En Sénégambie méridionale, cette période correspond aux trois premiers mois de la saison sèche, c'est-à-dire entre le début du mois de novembre et la fin du mois de décembre (tableau 1).

Tableau 1 - Dates d'acquisition et caractéristiques des données images utilisées

\begin{tabular}{|l|l|l|l|}
\hline 1972 & 1988 & 2000 & 2016 \\
\hline MSS & TM & ETM+ & OLI \\
Résolution :60 m & Résolution : $30 \mathrm{~m}$ & Résolution : $30 \mathrm{~m}$ & Résolution : $30 \mathrm{~m}$ \\
\hline
\end{tabular}




\begin{tabular}{|c|c|c|c|}
\hline $\begin{array}{l}\text { Path : } 220 \\
\text { Row : } 051 \\
5 \text { Novembre }\end{array}$ & $\begin{array}{l}\text { Path : } 205 \\
\text { Row : } 051 \\
29 \text { Novembre }\end{array}$ & $\begin{array}{l}\text { Path : } 205 \\
\text { Row : } 051 \\
8 \text { Décembre }\end{array}$ & $\begin{array}{l}\text { Path : } 205 \\
\text { Row : } 051 \\
26 \text { Novembre }\end{array}$ \\
\hline Path : 219 & Path : 204 & Path : 204 & Path : 204 \\
\hline Row : 051 & Row : 051 & Row : 051 & Row : 051 \\
\hline 4 Novembre & 8 Décembre & 1 Décembre & 19 Novembre \\
\hline
\end{tabular}

\section{Les prétraitements}

7 Les prétraitements se résument ici aux opérations de redressement des images et de "mosaïquage" des scènes contigües.

\section{Redressement des images}

8 Comparer des images issues de deux capteurs différents représente une tâche ardue en raison à la fois de la différence de résolution des capteurs ${ }^{5}$, du nombre de lignes et de colonnes $^{6}$ et de l'étendue couverte d'une scène à l'autre. La superposition pixel à pixel des images implique à la fois une correction géométrique et un redimensionnement de l'ensemble des données-images afin de les rendre comparables (Zitova et Flusser, 2003 ; Joly, 1987).

\section{Ré-échantillonnage et mosaïquage des images}

9 Le ré-échantillonnage consiste à affecter les caractéristiques numériques et spatiales des pixels de l'une des images prise comme référence (image maîtresse) à l'ensemble des autres images (images esclaves) : Ici, c'est la scène la plus récente (2016) (illustration $2 b$ ) qui a été l'image maîtresse pour les images prises aux dates antérieures (1972, 1988, 2000) (illustration 2a). Cette opération n'est pas sans conséquence sur les images résultant du traitement. En effet, passer d'une résolution de $80 \mathrm{~m}$ à $30 \mathrm{~m}$ ou l'inverse fait entraîne inexorablement une perte d'informations, ce qui peut avoir des répercussions sur la cartographie des changements (Soumaré et al., 2020). Le choix de l'image de 2016 comme référence se justifie par la volonté de réduire au maximum la perte d'information puisque seule l'image de 1972 possède une résolution spatiale différente des autres. Ici, la référence géographique n'intervient pas dans le calcul, seules les dimensions du pixel et l'étendue totale de la scène étant prises en compte. C'est ce qui fait d'ailleurs que les pixels des images ainsi crées par ré-échantillonnage possèdent les mêmes dimensions (illustration 2c). Après avoir subi la série de corrections évoquées précédemment, les scènes (est et ouest) sont utilisées pour composer une mosaïque constituant une couverture continue et totale de la Sénégambie méridionale (illustration 3). 
Illustration 2a - Image MSS du 4 novembre 1972 (image esclave)

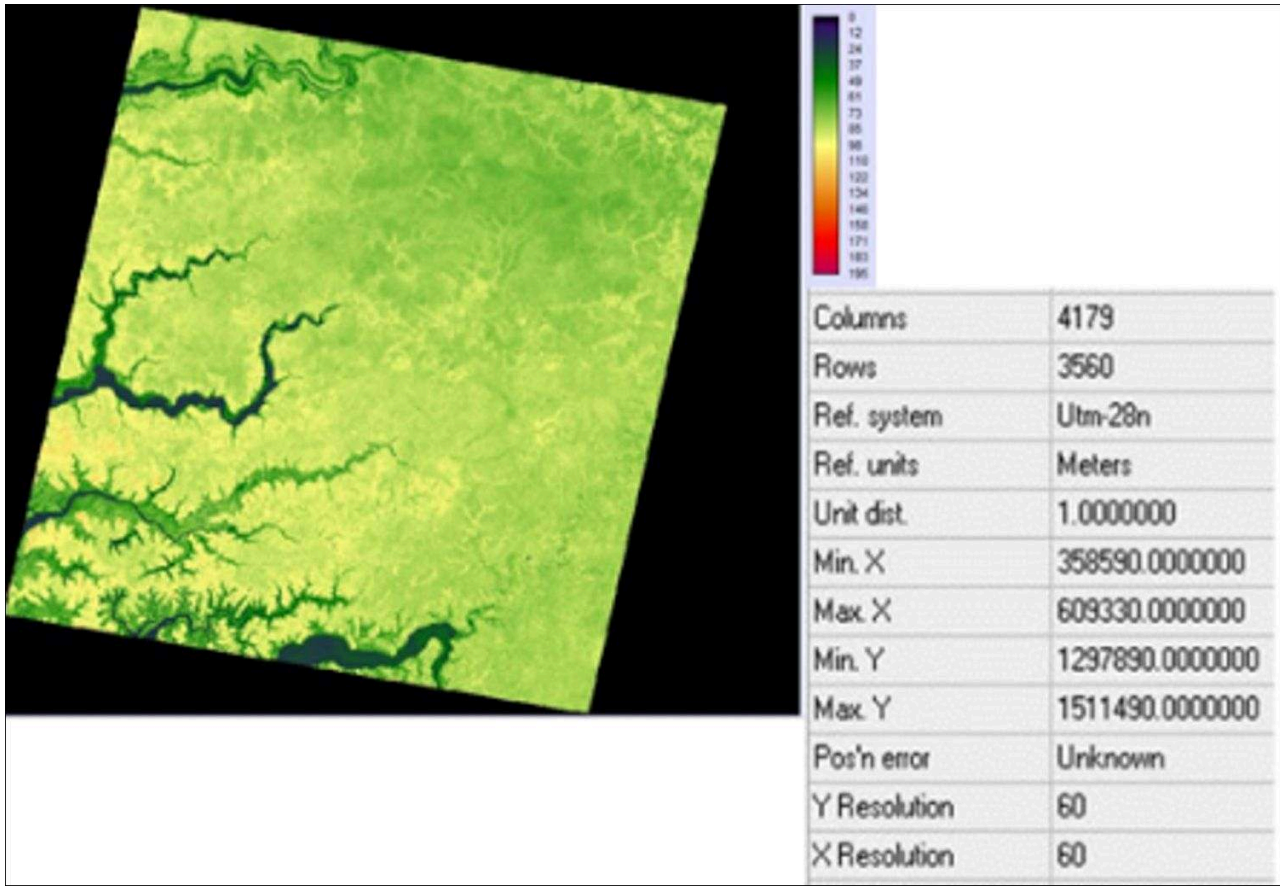

Illustration 2b - Image OLI du 11 novembre 2016 (image maitresse)

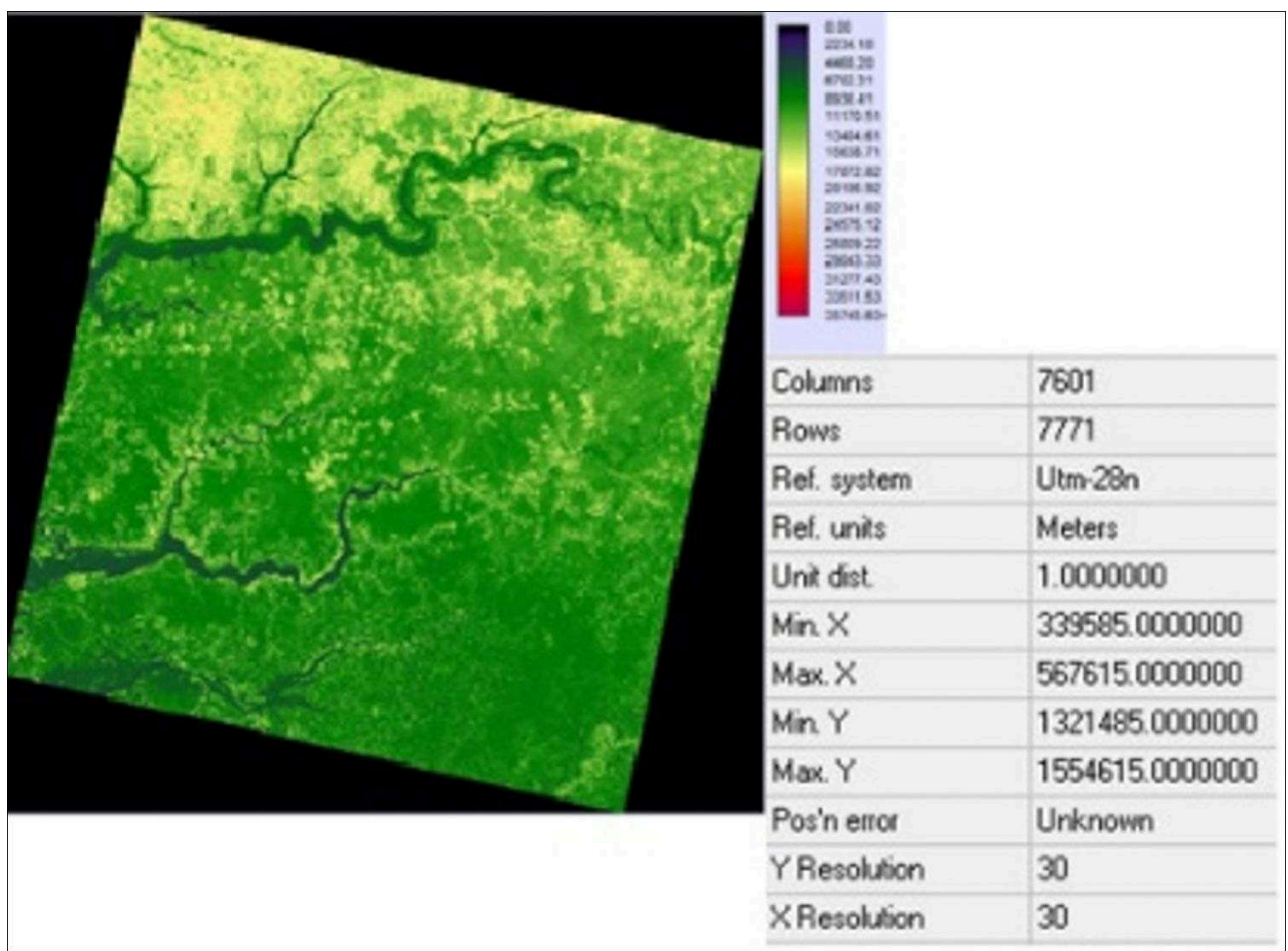


Illustration 2c - Image MSS du 4 novembre 1972 après le ré-échantillonnage

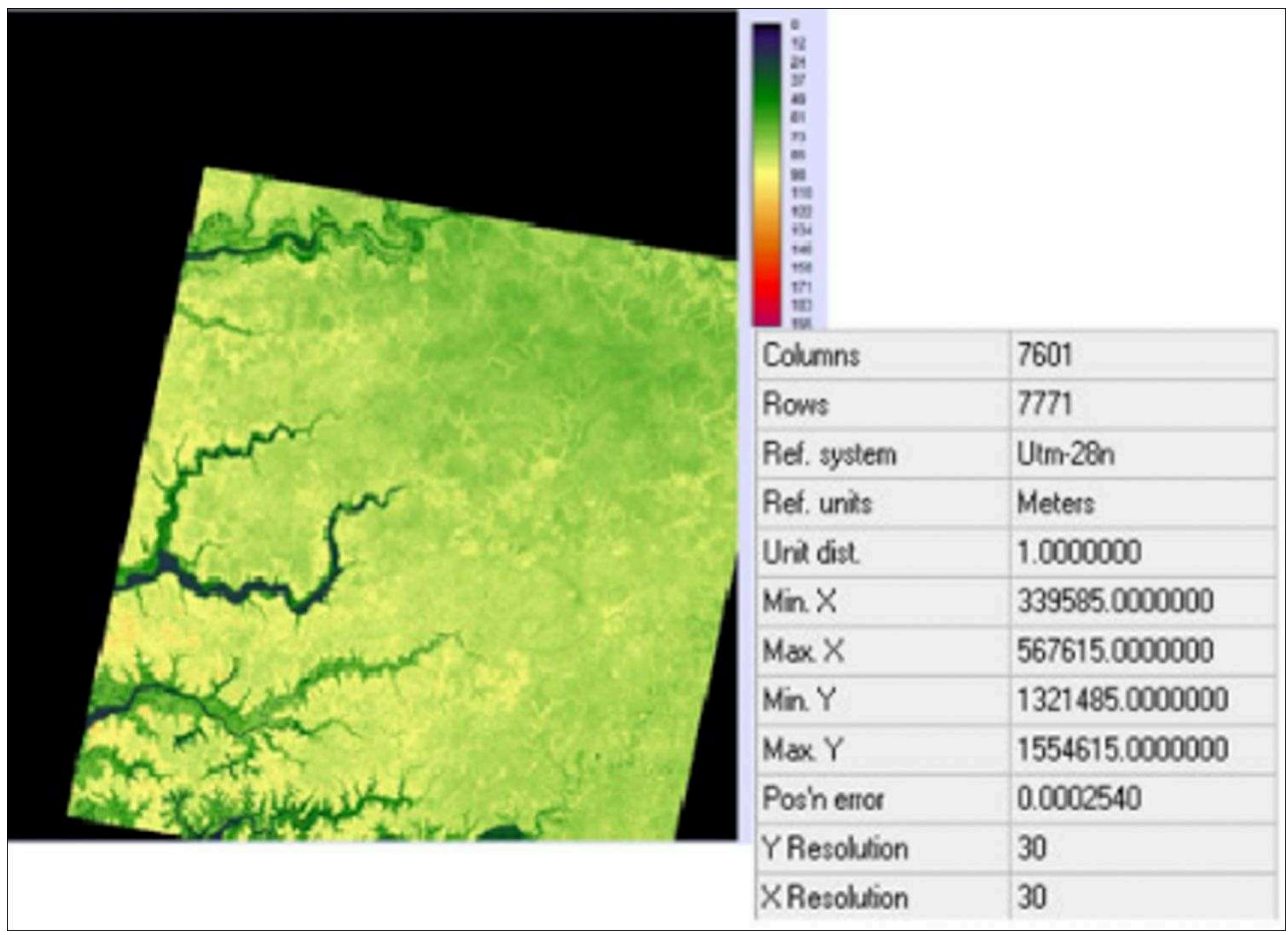

Illustration 3 - Composition colorée en fausses couleurs de la mosaïque de novembre 2016

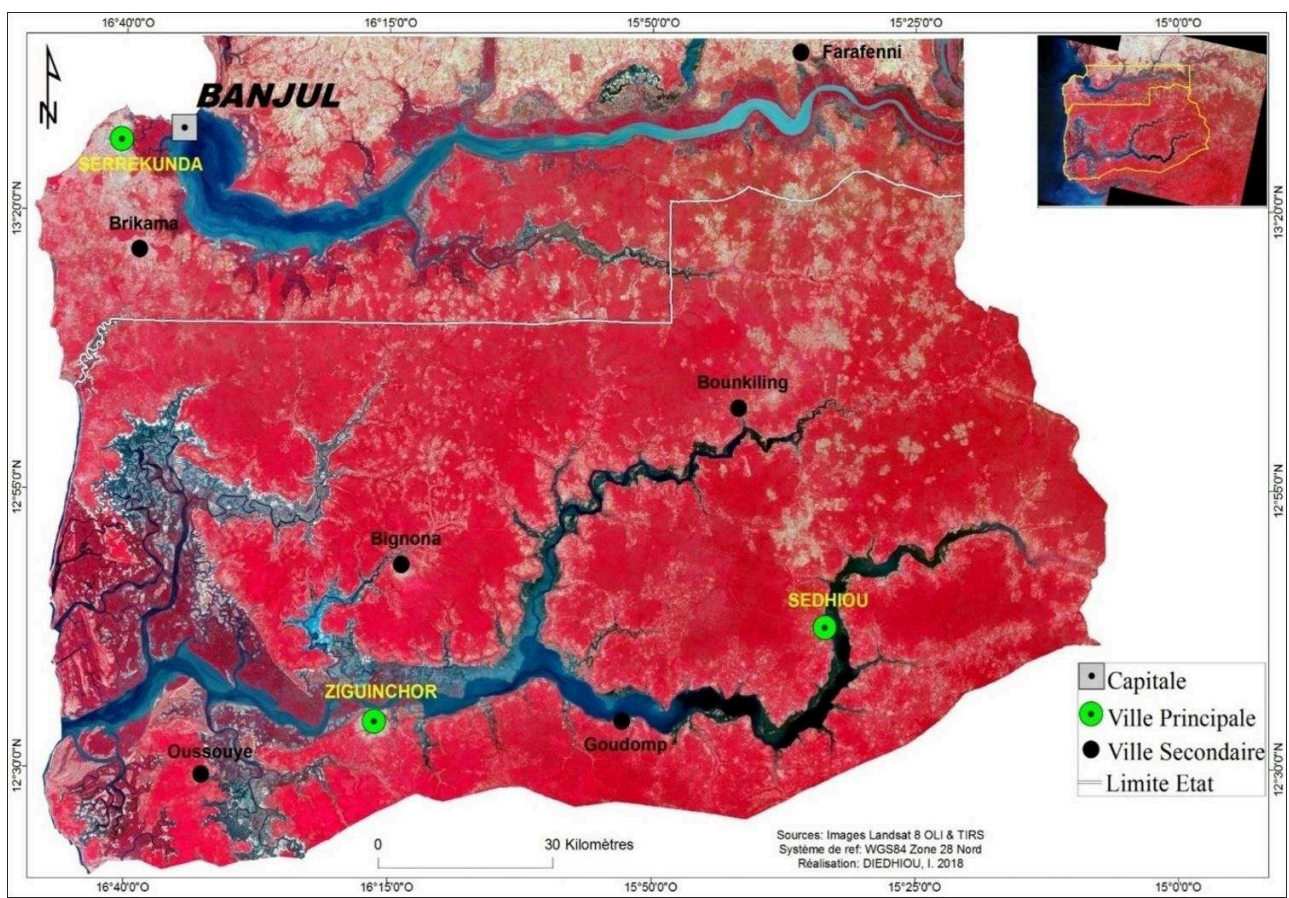

\section{Approche méthodologique}

10 Si les critères d'analyse comme la teinte, la texture et la structure sont encore utilisés pour identifier visuellement les objets présents sur une scène, la valeur spectrale des pixels reste le critère principal pour les classer par traitement numérique (Lawrence et 
al., 2010 ; Tang, et al., 2013). L'intérêt de ce type de classification multispectrale appelée «pixel à pixel » réside dans le fait qu'elle permet de rester proche de l'information originelle contenue dans l'image (Le Hégarat-Mascle, 2003). La méthodologie mise en place est schématisée sur l'illustration 4 .

\section{Choix de l'approche pour la cartographie de l'occupation du sol}

La méthode choisie est celle de la classification non supervisée reposant sur l'algorithme des Nuées Dynamiques avec tirage aléatoire des centres initiaux (Diday, 1971). Cette méthode a souvent été utilisée en Télédétection sous la dénomination de Kmoyennes dans le cadre d'une démarche de classification multispectrale non supervisée notamment par Andrieu et Mering (2008) et par Solly et ses collaborateurs (2020). Ici à l'instar de ce qu'ont fait (Oswald et al., 2010) pour reconstituer les changements des états de la végétation de Maçaranduba dans l'État de Para, au Brésil entre 1997 et 2006, nous avons adopté une approche rétrospective afin d'identifier les différentes catégories de l'occupation du sol devant figurer sur les cartes: pour y parvenir, le comportement spectral des classes thématiques dites de "référence " provenant d'une carte récente a été comparé avec celui des classes générées par la classification non supervisée des images prises aux autres dates.

Illustration 4 - Séquence des étapes de traitement

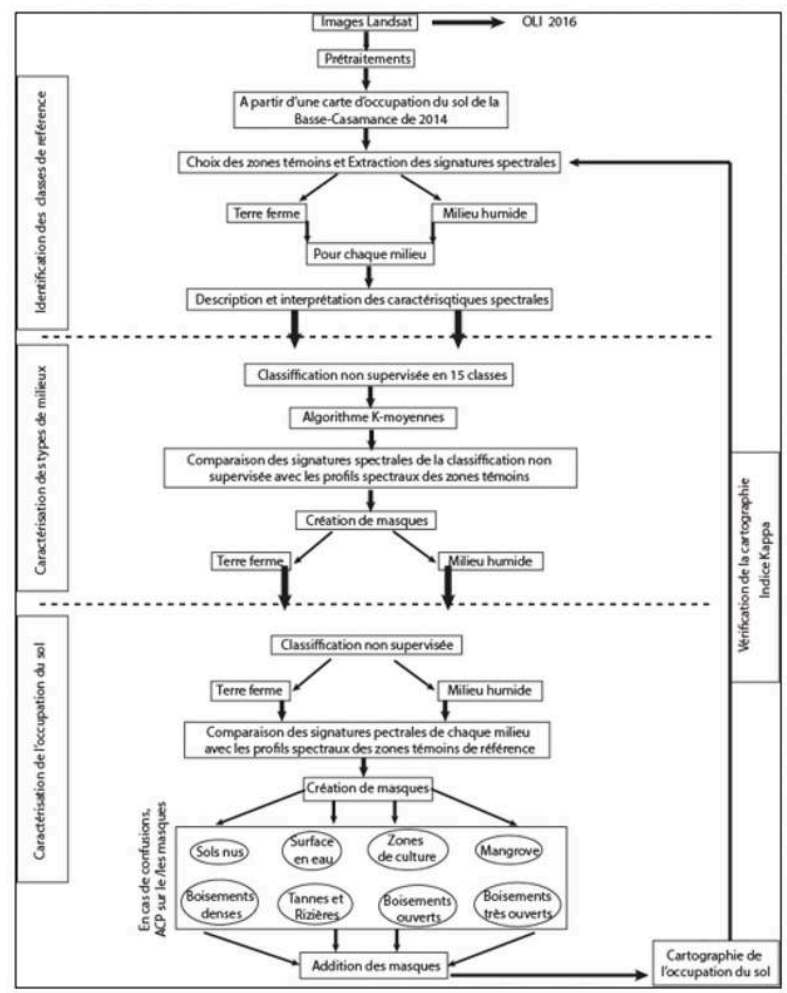

\section{Postulat de base de l'approche}

En partant du principe selon lequel chaque objet à la surface de la terre possède une signature spectrale qui lui est propre, on peut envisager de cartographier l'occupation du sol d'une région à des dates antérieures, en se basant sur les signatures spectrales 
caractérisant les états de surface telles qu'elles ont été extraites à partir d'une scène prise à une date où l'on dispose de réalités de terrain associées à ces états de surface. Dans ce type d'approche, l'un des facteurs majeurs à prendre en compte est la saisonnalité. Ainsi, lorsqu'on cherche à comparer les états de surfaces entre deux dates éloignées de plusieurs années, les images doivent être acquises au cours de la même période de l'année car la réponse spectrale d'un même type de surface varie en fonction des saisons et des conditions météorologiques lors de la prise de vue.

\section{Identification des classes à cartographier}

13 L'interprétation des classes issues de la classification multispectrale non supervisée suivant une nomenclature thématique préétablie se fait généralement par le biais de l'analyse des comportements spectraux de ces classes. Cette démarche exige donc d'avoir une référence à partir de laquelle les profils spectraux des classes thématiques que l'on cherche à identifier seront extraits. Ici, la référence est une carte de l'occupation du sol de la Basse Casamance en 201 (illustration 5) conçue par T. Sané dans le cadre de ses travaux de thèse (Sané, 2017). Le choix de cette référence se justifie parce que les différentes catégories de paysages de Sénégambie méridionale que l'on cherche à cartographier ici sont représentés de façon exhaustive en Basse Casamance. De plus le niveau de détail élevé ( 15 classes), le caractère récent et la précision des informations figurant sur la carte qui témoignent d'un important travail de relevés de terrain réalisé en amont légitiment ce choix. Notre objectif étant de caractériser les couverts forestiers selon leur densité et leur évolution durant la période étudiée, nous n'avons pas conservé le niveau de détail de la carte de T. Sané (2017). Aussi, cherchant essentiellement à différencier les formations boisées et les autres types de couverture du sol sur terre ferme d'une part, la mangrove des surfaces non boisées en milieu humide d'autre part, nous avons fusionné entre elles certaines des 15 classes d'occupation du sol issues de la carte de référence, pour n'en retenir que huit. Ces choix sont explicités dans le tableau 2 où figurent également les codes numériques qui seront attribués aux classes thématiques que nous cherchons à représenter.

Tableau 2 - Typologie retenue pour la cartographie de l'occupation du sol aux différentes dates

\begin{tabular}{|l|l|l|l|}
\hline \multicolumn{2}{|l|}{ MILIEU HUMIDE } & \multicolumn{2}{l|}{ TERRE FERME } \\
\hline 1 & Surfaces en eau & 4 & Sols nus (habitat et sol nu / plage) \\
\hline 2 & $\begin{array}{l}\text { Formations de mangrove (mangroves ouvertes } \\
\text { et mangroves denses) }\end{array}$ & 5 & Espaces cultivés \\
\hline 3 & Tannes et rizières (tannes, vasières, rizières) & 6 & $\begin{array}{l}\text { Boisements très ouverts (savanes arborées et } \\
\text { arbustives) }\end{array}$ \\
\hline & 7 & $\begin{array}{l}\text { Boisements ouverts (forêts claires et savanes } \\
\text { boisées) }\end{array}$ \\
\cline { 2 - 4 } & 8 & $\begin{array}{l}\text { Boisements denses (plantations forestières, } \\
\text { forêts galeries et forêts denses) }\end{array}$ \\
\hline
\end{tabular}


Illustration 5 - : Occupation du sol de la Basse Casamance en 2014

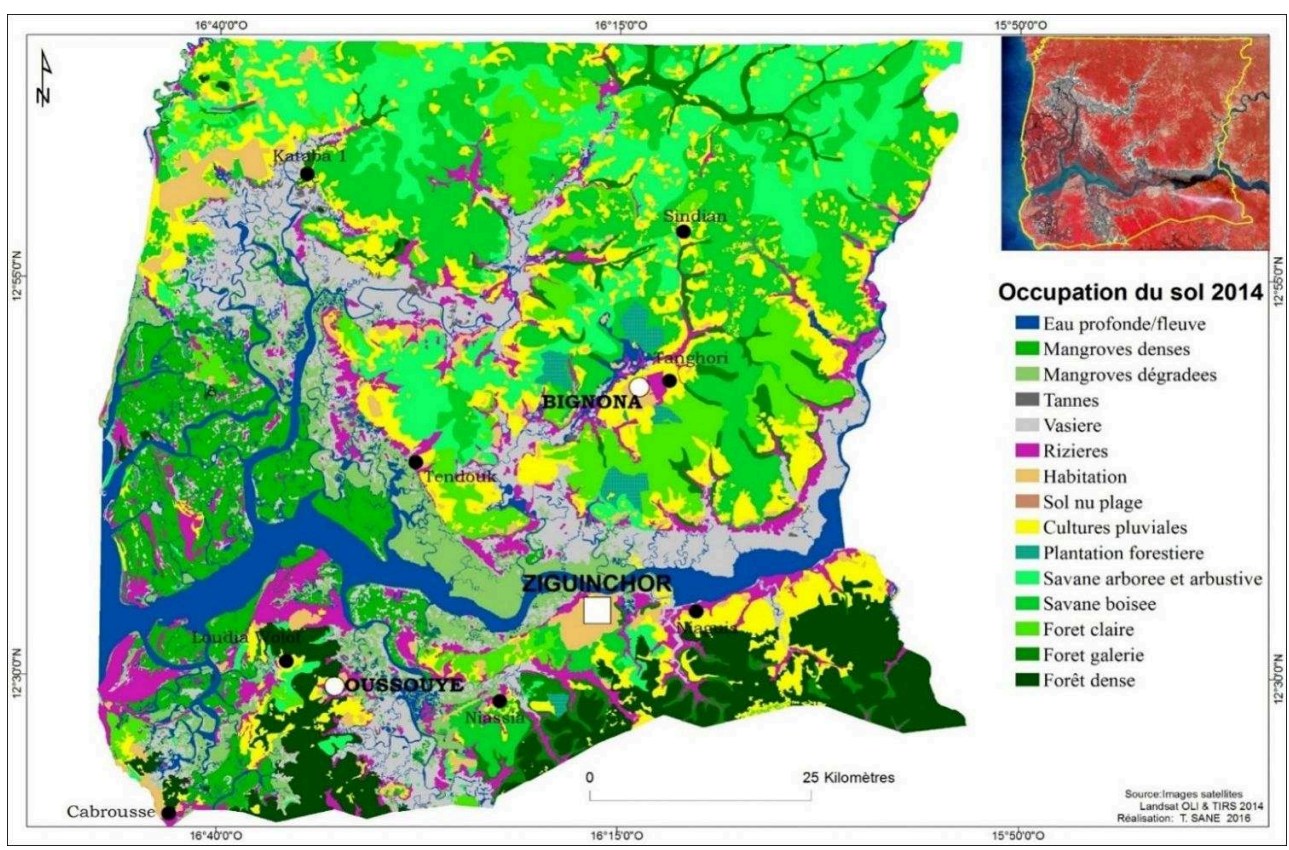

Source : Sané, 2017.

\section{Choix des zones témoins et extraction des profils spectraux de référence}

Deux critères ont été retenus dans le choix des zones témoins: d'une part obtenir le plus large éventail possible de profils spectraux et d'autre part pouvoir prendre en compte les différentes catégories d'occupation du sol présentes au sein de la zone d'étude, telles qu'elles sont listées sur le tableau 2. En outre, pour cartographier des paysages aussi complexes que ceux de la Sénégambie, une classification séparée par catégorie de milieu, humide ou de terre ferme, nous a paru propre à limiter les risques de confusion entre des surfaces ayant des réponses spectrales relativement similaires situées dans des milieux différents comme c'est le cas des tannes vifs qui se trouvent en milieu humide et des sols nus situés sur terre ferme ${ }^{7}$. Ainsi, chaque zone témoin choisie sur la carte d'occupation du sol de 2014 (illustration 6) et sur la composition colorée de la même année (illustration 7), est identifiée par un code selon le type de milieu (numérique pour les paysages de terre ferme et alphabétique pour ceux en milieu humide). 
Illustration 6 - Localisation des zones témoins sur la carte d'occupation du sol de 2014 (Basse Casamance)

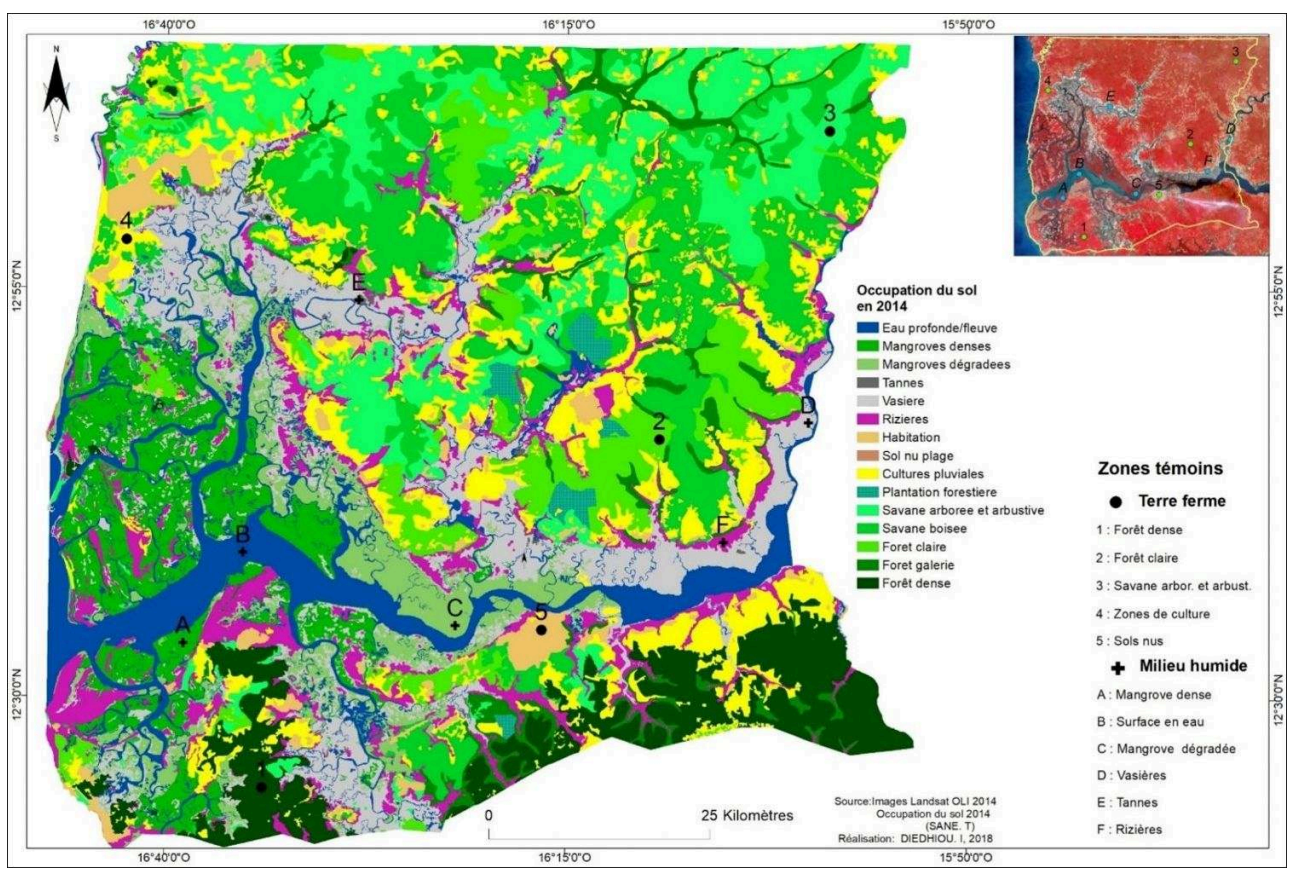

Illustration 7 - Localisation des zones témoins sur la composition colorée en fausses couleurs de 2014 (Basse Casamance)

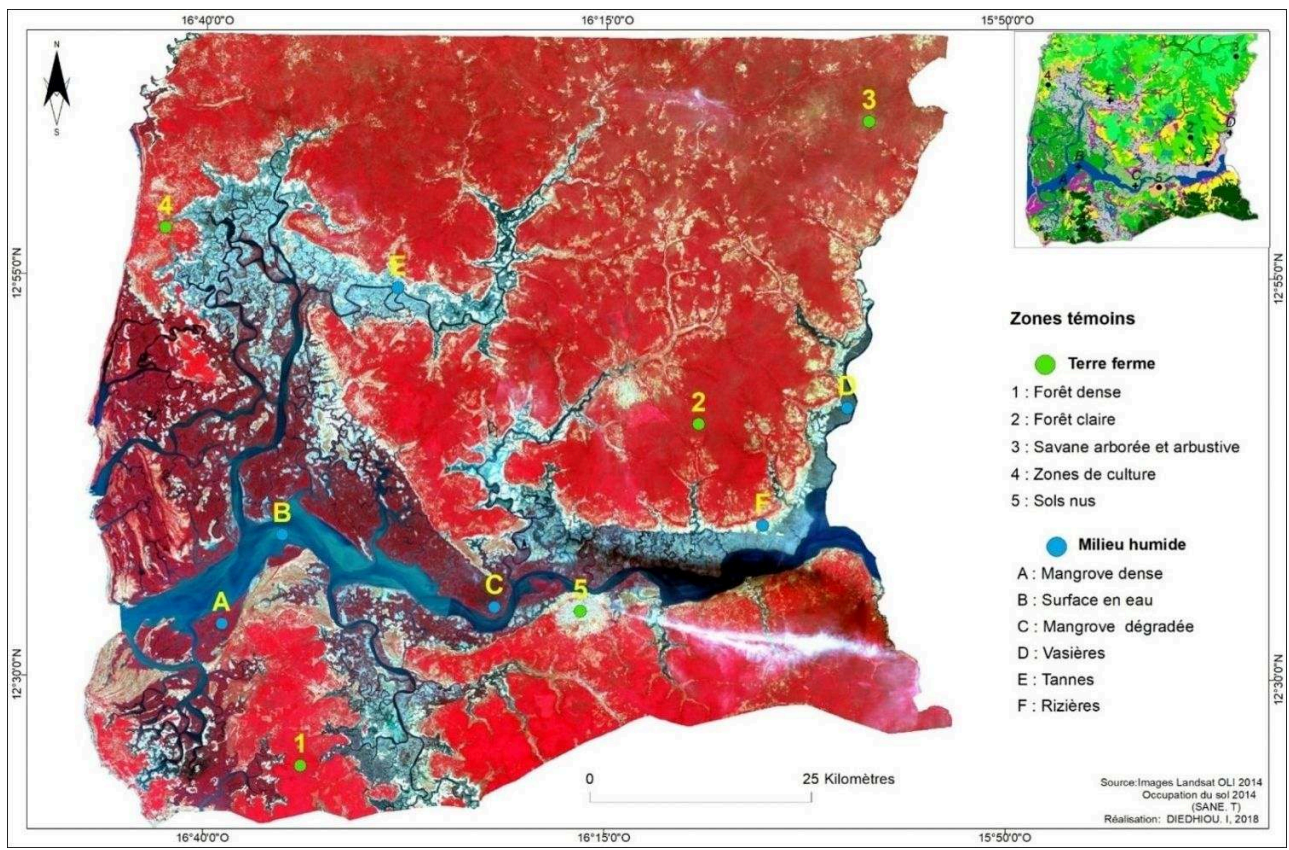

15 Une fois générée, la signature de chaque zone témoin est analysée afin de faciliter ultérieurement le travail de reconnaissance des catégories d'occupation du sol d'après le comportement spectral des différentes classes issues de la classification multispectrale réalisée sur l'une ou l'autre des images sélectionnées pour cette étude. 


\section{Description et interprétation des caractéristiques spectrales des surfaces de terre} ferme

16 L'analyse des profils de luminance des zones témoins situées sur terre ferme (illustration 8) fait ressortir deux grandes catégories de réponses spectrales : d'une part des surfaces caractérisées par des pics d'intensité variable dans le proche infrarouge, une luminance plus ou moins faible dans le visible et l'infrarouge moyen, et d'autre part, celles dont la luminance augmente graduellement du visible à l'infrarouge moyen. De manière générale, la description de la première catégorie de surface correspond au comportement type de la végétation, qui peut varier selon la structure du couvert. Lorsque celui-ci est dense ou se trouve associé à d'autres plantes à forte activité chlorophyllienne comme les plantations forestières (échantillon 1), la luminance est importante dans le proche infrarouge et faible dans les bandes visible et moyen infrarouge du spectre. Pour l'échantillon 2, le pic de luminance dans le proche infrarouge est sensiblement moins élevé ce qui pourrait s'expliquer par le fait que la réponse spectrale des groupements d'arbres a pu être influencée par l'existence de traces de brulis au sol qui atténuent la luminance moyenne dans le proche infrarouge des surfaces végétalisées.

Illustration 8 - Signatures des zones échantillons en milieu de terre ferme

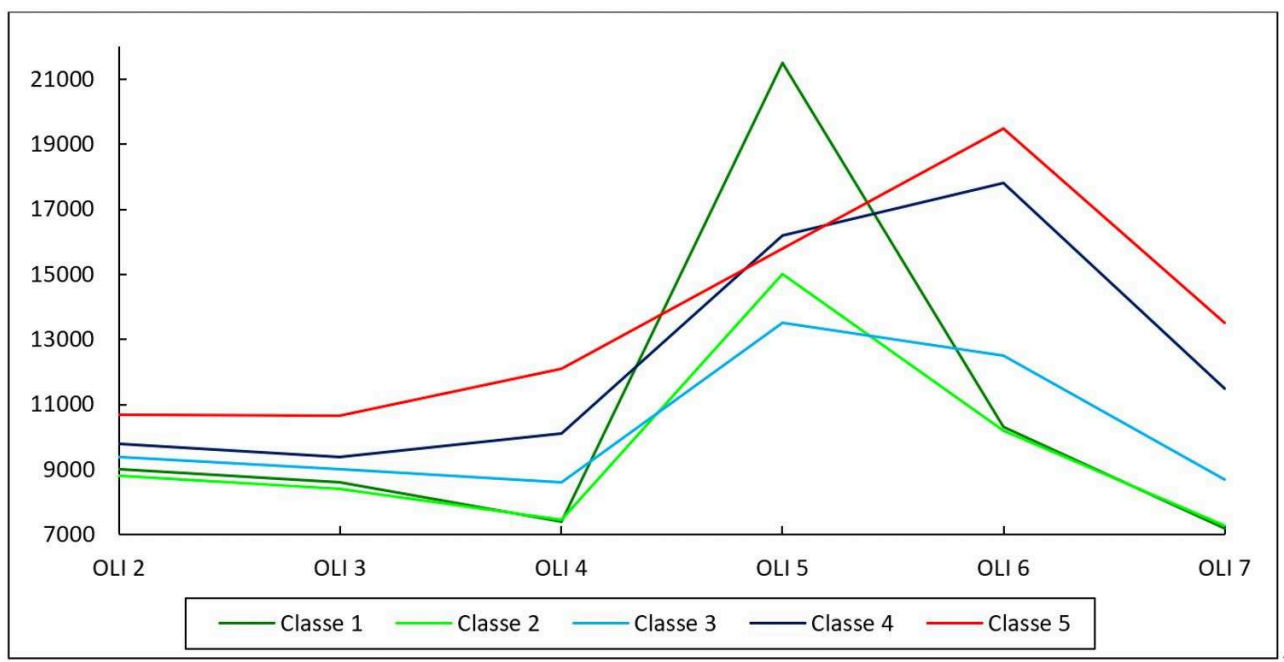

L'échantillon 2 dont il est question ici correspond vraisemblablement à des surfaces boisées exposées à des feux de brousse répétés chaque année, et qui seraient en partie à l'origine de l'ouverture de nombreux boisements de la rive nord de la Casamance. Dans tous les cas, les faibles luminances dans le visible et le moyen infrarouge associés à un pic dans proche infrarouge nous conduit à considérer ce type de boisement comme étant ouvert.

Lorsque la couverture végétale ouverte est associée à un sous-bois clairsemé (échantillon 3), l'alternance entre sol nu et couvert végétal produit une signature alliant certaines des caractéristiques du sol nu (forte réponse dans le visible et dans le moyen infra rouge) et de la végétation (forte luminance dans le proche infrarouge). En zone tropicale, ce type de comportement spectral correspond à une formation végétale très ouverte, car la réponse spectrale de la surface est affectée par la réflectance des sols à cause de la faible couverture végétale (Devineau, 1990). Le profil radiométrique 
de ce type de surfaces se présente alors avec deux valeurs hautes de luminance successives respectivement dans le proche infrarouge à cause d'un recouvrement modeste du couvert végétal et dans le moyen infrarouge en raison de la faible teneur en eau des sols en saison sèche.

Le profil de l'échantillon 4 peut être associé à des surfaces peu végétalisées dont la teneur en eau est faible comme c'est le cas des zones cultivées en saison sèche.

Pour l'échantillon 5, le profil spectral montre une luminance qui croît progressivement du visible à l'infrarouge moyen. Contrairement, aux autres types de surface, le profil de cette zone échantillon est celui d'une surface claire, dépourvue de végétation, d'où sa forte luminance dans le visible, l'absence de pic dans le proche infrarouge et un pic de luminance dans le moyen infrarouge du fait de sa très faible teneur en eau. C'est la réponse spectrale typique des surfaces dépourvues de pigments foliaires qui, au sein des paysages de terre ferme, correspondent à des sols nus clairs et secs (tableau 3

Tableau 3 - Correspondance entre zones échantillons et surfaces de terre ferme

\begin{tabular}{|l|l|l|}
\hline Zones témoins & Boisements ouverts $=2$ & Zones de culture $=4$ \\
\hline Boisements denses =1 & Boisements très ouverts =3 & Sols nus $=5$ \\
\hline
\end{tabular}

\section{Description et interprétation des caractéristiques spectrales des surfaces en zone} humide

En milieu humide (illustration 9), on distingue une catégorie de surfaces représentée par l'échantillon $\mathrm{E}$ dont le profil se démarque des autres aussi bien par une augmentation rapide et continue des valeurs de luminance en allant du visible au proche infrarouge que par un important pic de luminance dans l'infrarouge moyen. Ce type de réponse présente des caractéristiques relativement semblables à celle des sols nus de terre ferme à la différence près que ces surfaces considérées, bien que situées en zone humide, ont souvent un pouvoir réfléchissant encore supérieur : il s'agit ici de tannes vifs'. À l'opposé, on remarque un type de profil où les valeurs de luminance diminuent graduellement avec l'augmentation des longueurs d'onde. Ce comportement spectral, est caractéristique des surfaces en eau. 
Illustration 9 - Signatures spectrales des zones échantillons en milieu humide

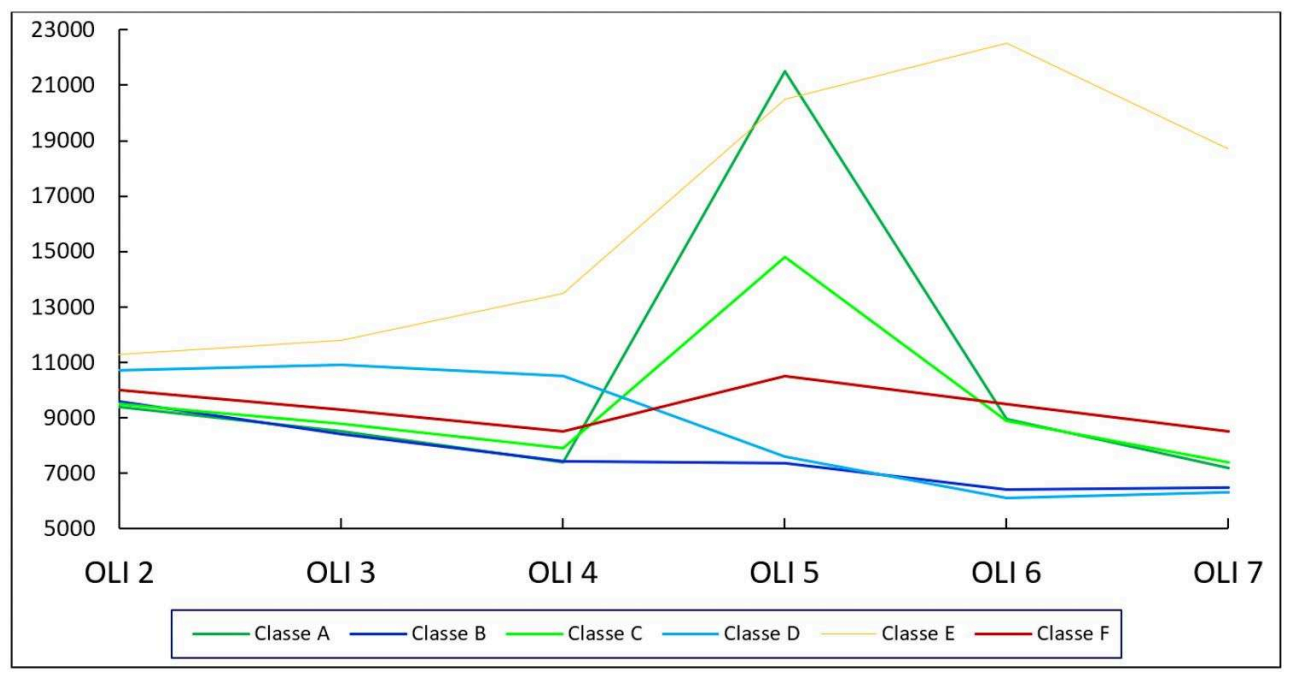

Il faut toutefois noter que si l'eau est turbide ou très peu profonde, la luminance est élevée dans les longueurs d'onde du visible ce qui donne aux surfaces correspondantes une teinte claire bien différente de celle de l'eau profonde sur la composition colorée. Ici, la zone témoin $\mathrm{D}$ dont le comportement spectral est celui de l'eau peu profonde correspond à celui de surfaces régulièrement recouvertes d'une pellicule d'eau comme le sont les tannes humides et les vasières. En revanche, le profil de l'échantillon $B$, où la luminance décroit continument du visible vers le proche et moyen infrarouge, est caractéristique du comportement spectral de l'eau profonde.

La dernière catégorie de profils spectraux est celle des surfaces végétalisées à l'intérieur desquelles on distingue, selon leur densité ou l'état du couvert, trois sousensembles: les boisements de mangrove denses (échantillon A), les boisements de mangrove ouvertes (échantillon C) et les rizières (échantillon F) (tableau 4).

Tableau 4 - Correspondance des zones échantillons avec les surfaces des zones humides

\begin{tabular}{|l|l|l|}
\hline Mangrove dense= A & Mangrove ouverte = C & Rizières= F \\
\hline Vasières = D & Surfaces en eau = B & Tannes = E \\
\hline
\end{tabular}

\section{Caractérisation des deux types de milieux : la terre ferme et le milieu humide}

L'objectif de cette première classification non supervisée en 15 classes $^{9}$ réalisée sur la totalité de la zone d'étude à partir de la scène Landsat OLI de novembre 2016 (illustration 10) est de différencier les deux catégories de milieux de la région : la terre ferme et le milieu humide. 
Illustration 10 - Classification non supervisée en 15 Classes Images du 11 et 26 novembre 2016

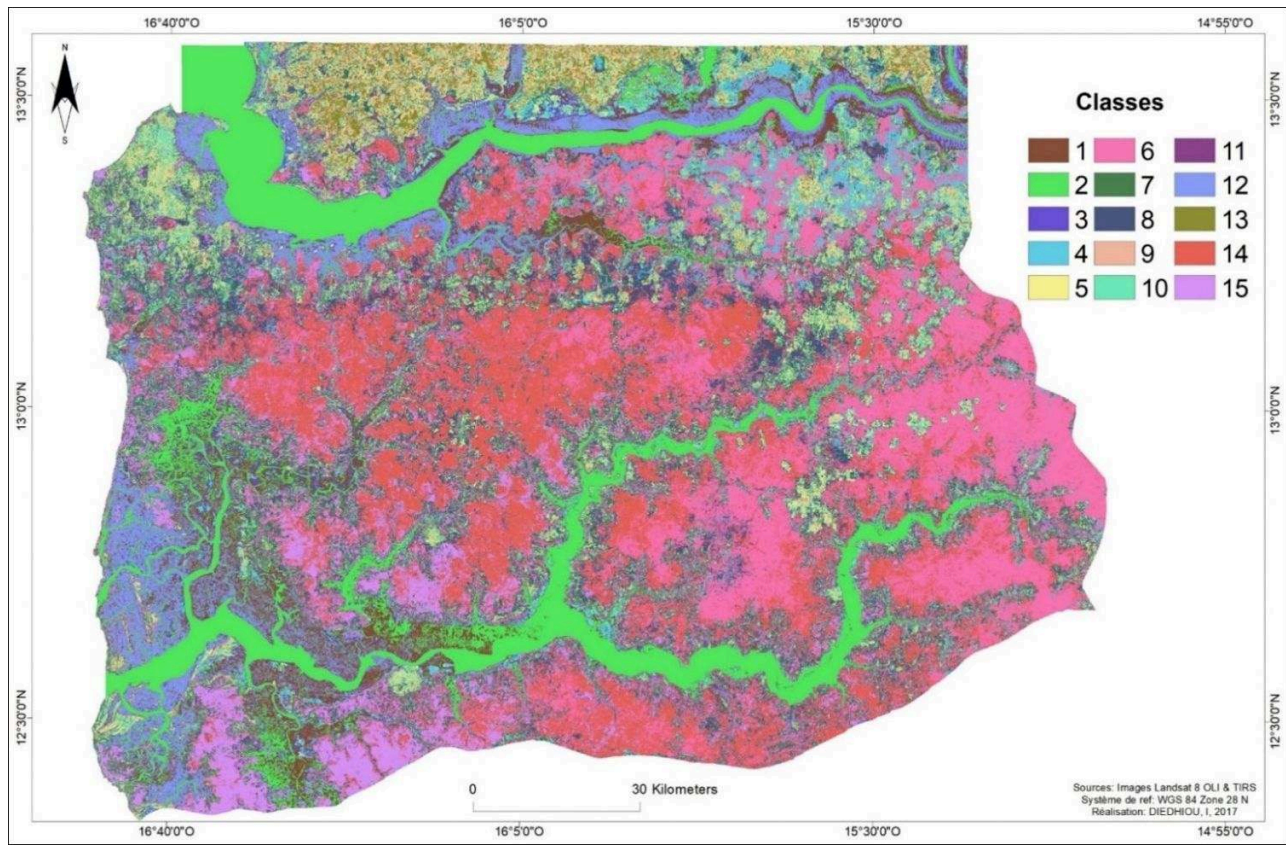

L'analyse des signatures spectrales résultant de la classification non supervisée permet, par comparaison avec le profil spectral des échantillons représentant les classes thématiques, de différencier ces milieux (illustration 11).

Illustration 11 - Profils spectraux de la classification générale de la mosaïque (Sénégambie méridionale, 11 et 26 novembre 2016)

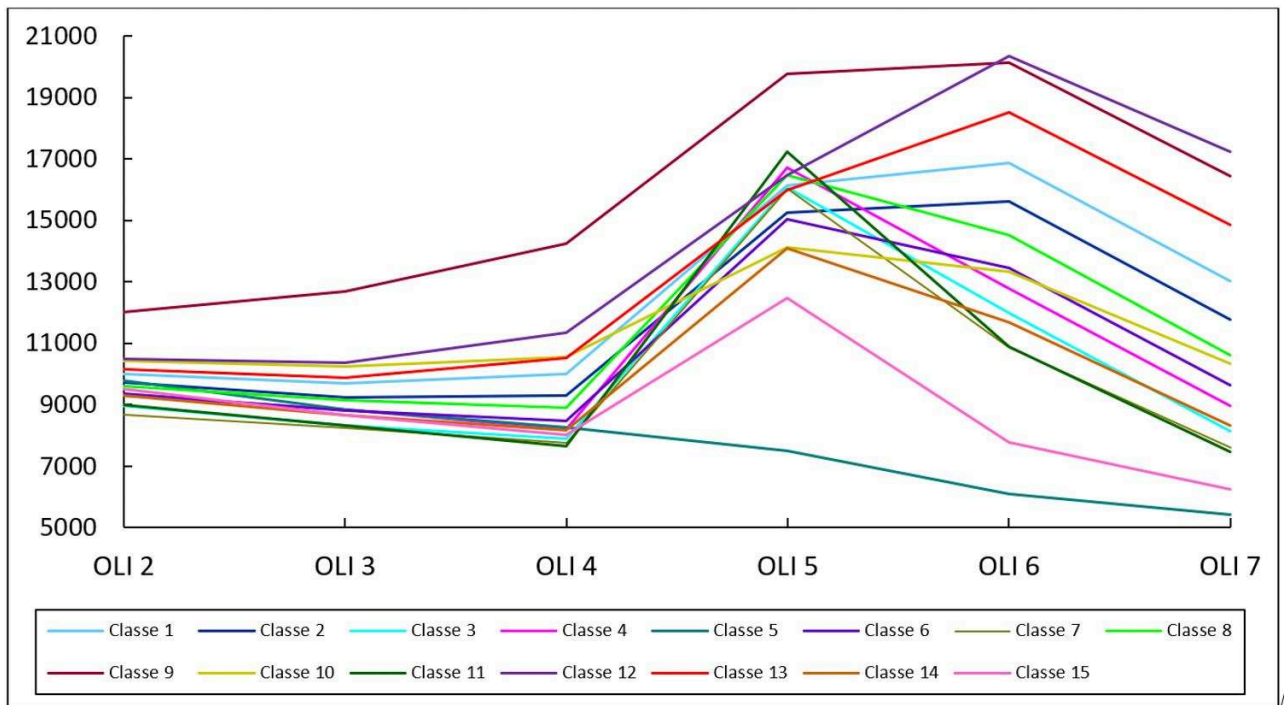

27 Les profils de luminance des deux milieux sont relativement contrastés notamment dans les grandes longueurs d'onde. En effet, dans les bandes infrarouges, les paysages de terre ferme ont la particularité d'être plus réfléchissants que celles situées en zones humides. Un tel pouvoir discriminant est dû ici à la fois à leur sensibilité à l'activité chlorophyllienne (dans le proche infrarouge) et à la teneur en eau (dans le moyen infrarouge). 
28 Ainsi toute surface présentant un important pic de luminance dans l'une des bandes de la partie infrarouge du spectre sera considérée comme appartenant aux paysages de terre ferme. Toutefois, si ce critère suffit généralement à distinguer les surfaces de terre ferme, nous avons parfois eu recours à l'interprétation visuelle de la composition colorée pour corriger les erreurs d'affectation ce qui a été le cas pour les tannes vifs dont la luminance peut être supérieure à celle des sols nus de terre ferme. De la même manière, lorsque la luminance est comparativement plus faible dans la partie infrarouge du spectre, les profils sont associés à des surfaces du milieu humide. Le tableau 5 résume l'affectation des 15 classes initiales à chacun des milieux considérés.

Tableau 5 - Reclassement des surfaces selon le type de milieu

\begin{tabular}{|c|c|c|c|}
\hline \multirow{3}{*}{ Milieu humide } & Classes $1 ; 3 ; 12$ & $=$ & Boisements de mangrove \\
\hline & Classe 2 & $=$ & Surfaces en eau \\
\hline & Classe 11 & $=$ & Tannes et / ou Rizières \\
\hline \multirow{5}{*}{ Terre ferme } & Classes $4 ; 5 ; 10$ & $=$ & Zones de Cultures \\
\hline & Classes $6 ; 14 ; 15$ & $=$ & Boisements denses \\
\hline & Classe 7 & $=$ & Boisements très ouverts \\
\hline & Classe 8 & $=$ & Boisements ouverts \\
\hline & Classes $9 ; 13$ & $=$ & Sols nus \\
\hline
\end{tabular}

Une fois isolé, chacun des milieux est considéré comme délimité de façon définitive, et servira de cadre de référence dans le traitement des dates antérieures ${ }^{10}$ (illustration 12). 
Illustration 12 - Les deux types de milieu en Sénégambie méridionale

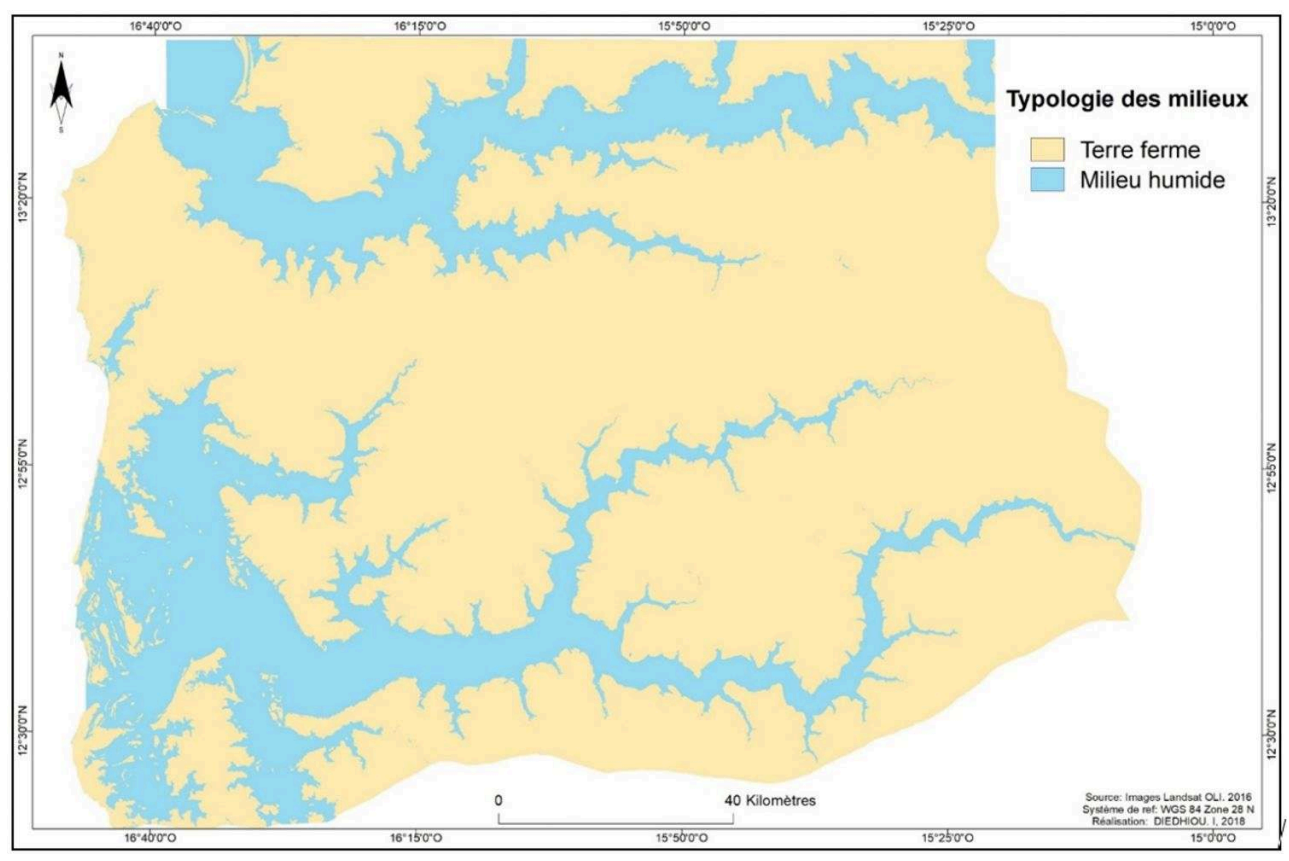

\section{Classifications non supervisées}

\section{Milieu humide}

L'analyse des profils spectraux des 15 classes au sein du milieu humide (illustration 13) met en évidence plusieurs catégories de surfaces qui, prises individuellement, révèlent des détails d'occupation du sol qui auraient été difficiles à déceler à l'aide d'une classification de la scène dans sa totalité. En nous référant aux profils spectraux des zones témoins en milieu humide (illustration 9 et tableau 4), la courbe de luminance qui se rapproche le plus de celle des rizières sur l'illustration 13 est celle de la classe 5 . Pour rappel, les surfaces rizicoles se caractérisent par une luminance non négligeable dans le visible, un faible pic de luminance dans le proche infrarouge et une diminution de la luminance dans l'infrarouge moyen. Contrairement aux autres surfaces végétalisées en milieu humide, les rizières s'identifient par leur faible teneur en pigments d'où leur réponse modeste dans le proche infrarouge.

31 L'analyse de la classification au sein du milieu humide a également permis de mettre en évidence deux autres classes : les classes 7 et 14 dont la signature spectrale, si elle est différente de celle qui a servi de référence pour reconnaître les rizières présentent toutefois des caractéristiques similaires à celles-ci. 
Illustration 13 - Classification non supervisée du milieu humide

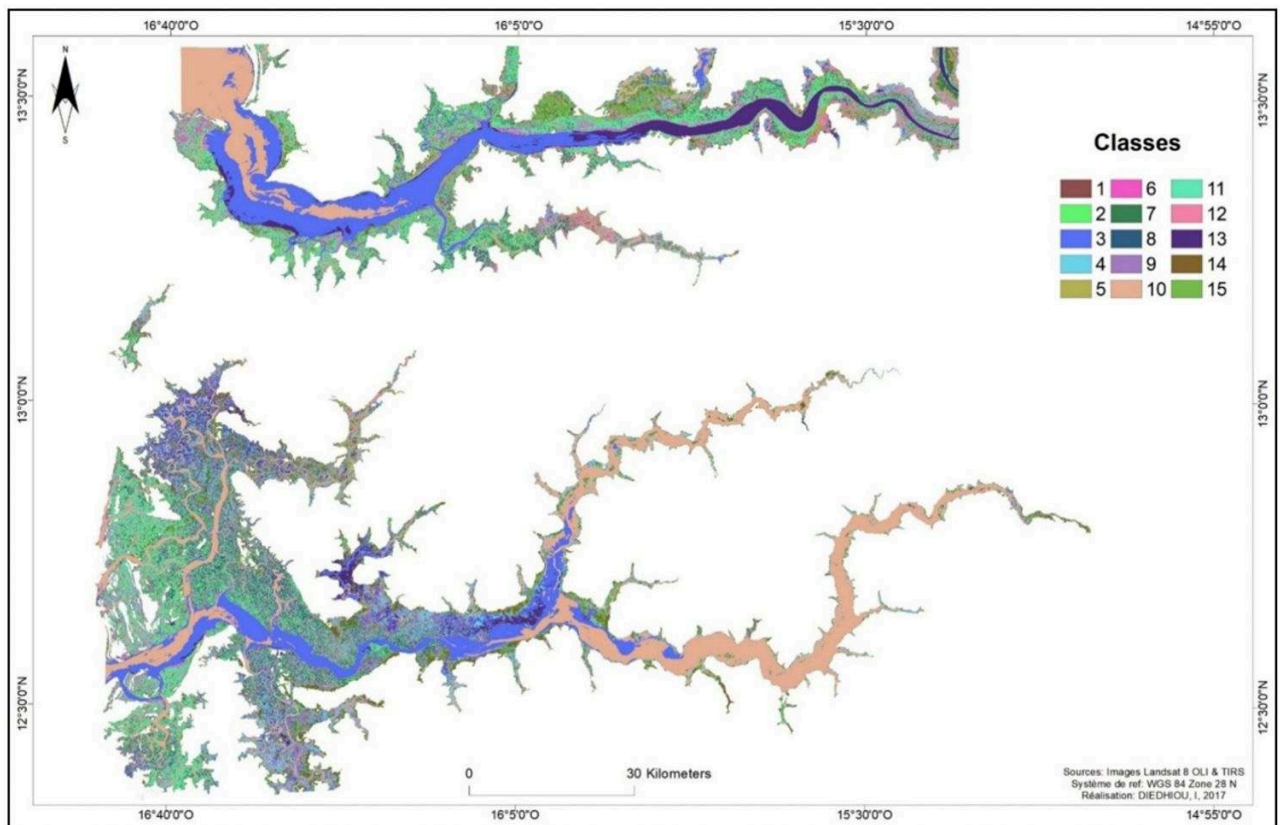

Illustration 14 - Profils spectraux de la classification non supervisée du milieu humide

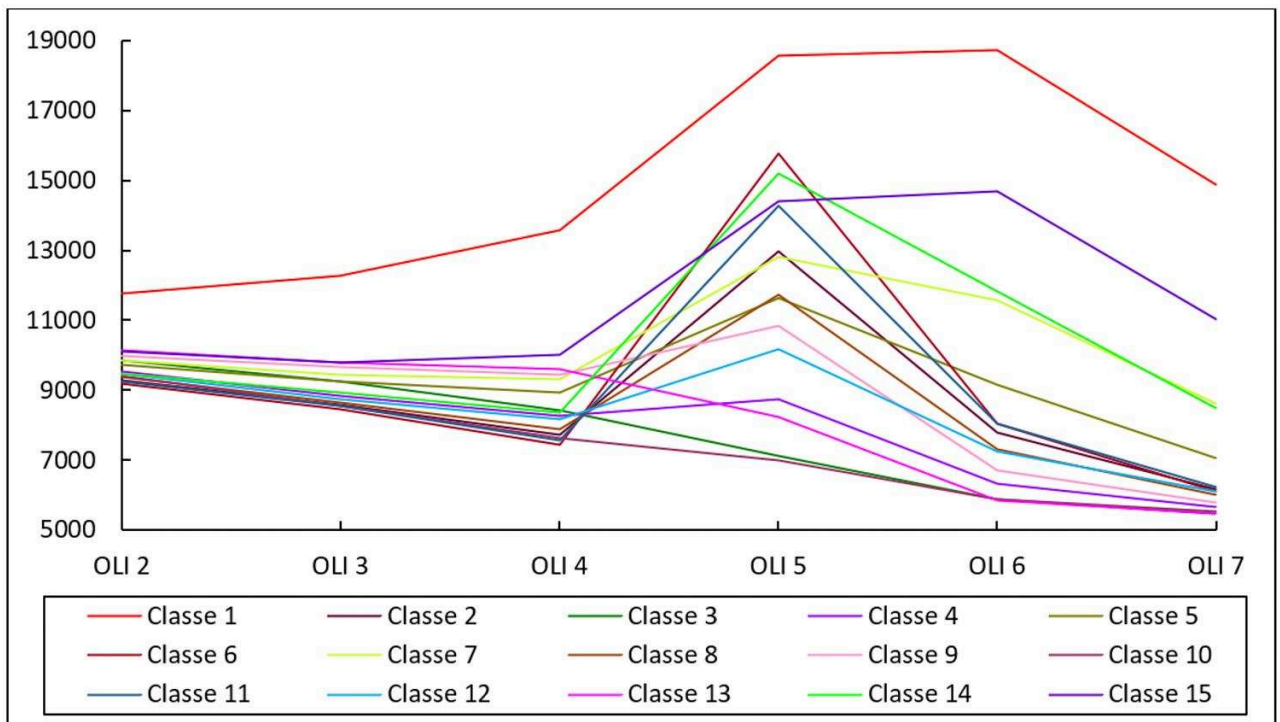

En effet en analysant les profils spectraux de ces classes dans le canal moyen infrarouge, on distingue deux catégories de rizières en fonction de leur teneur en eau : d'une part, celles des pentes représentées par les classes 7 et 14, et de l'autre, celles de bas-fonds dont la classes 5 est la parfaite illustration (illustration 14). Dans le même ordre d'idée, nous avons regroupé les classes 1 et 15 dont les profils spectraux sont similaires à celui des surfaces très peu ou pas végétalisées des zones humides comme les tannes (illustration 8) avec une luminance qui augmente progressivement du visible au moyen infrarouge.

Les profils des classes 1 et 15 doivent cependant être distingués. Les tannes nus et secs voire recouverts d'une croûte salée ont une très forte luminance dans toutes les longueurs d'onde du spectre. Le profil de la classe 1 est de ce type. Par contraste, le 
profil de la classe 15, caractérisé par de fortes luminances essentiellement dans le proche et moyen infrarouge, a permis d'interpréter cette classe comme étant des tannes herbus. De façon générale, dans ces marais maritimes, les unités paysagères s'agencent suivant la toposéquence suivante (illustration 15): les tannes nus secs voire salés se localisent dans les zones les plus élevées (classe 1) ; ils sont suivis par les tannes secs herbus (classe 15), puis par les rizières de plateau ${ }^{11}$ (classes 7 et 14 ) et enfin par les rizières de bas-fonds (classe 5).

Illustration 15 - Typologie des tannes et des rizières

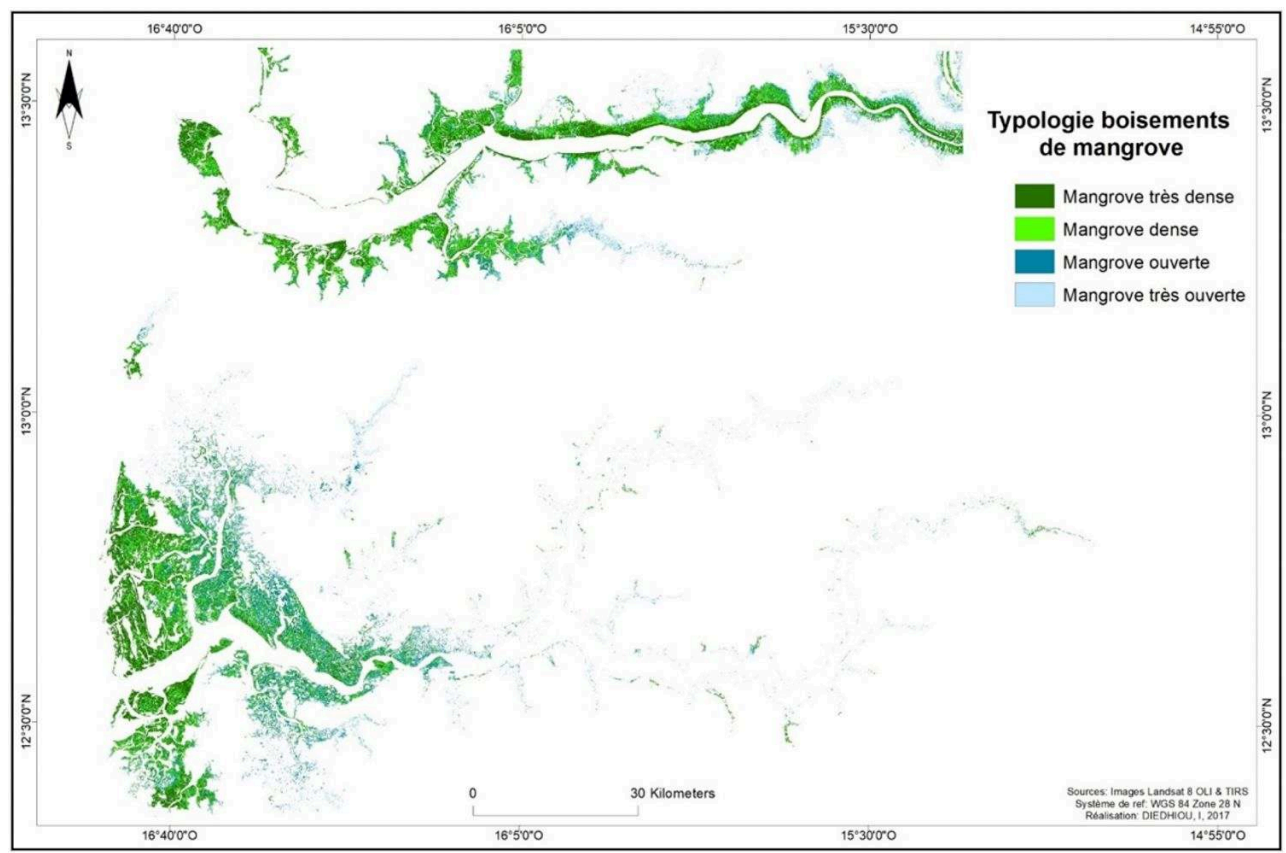

34 À l'opposé, les surfaces très humides ou couvertes d'eau sont facilement reconnaissables grâce à leur comportement spectral où la luminance diminue progressivement le long du spectre. Ces surfaces humides peuvent être divisées en trois catégories: les tannes humides (classes 13), les tannes (très humides) ou recouverts d'une lame d'eau assez haute (classe 3), et enfin l'eau profonde (classe 10) (illustration 16). 
Illustration 16 - Typologie des tannes humides et des surfaces en eau

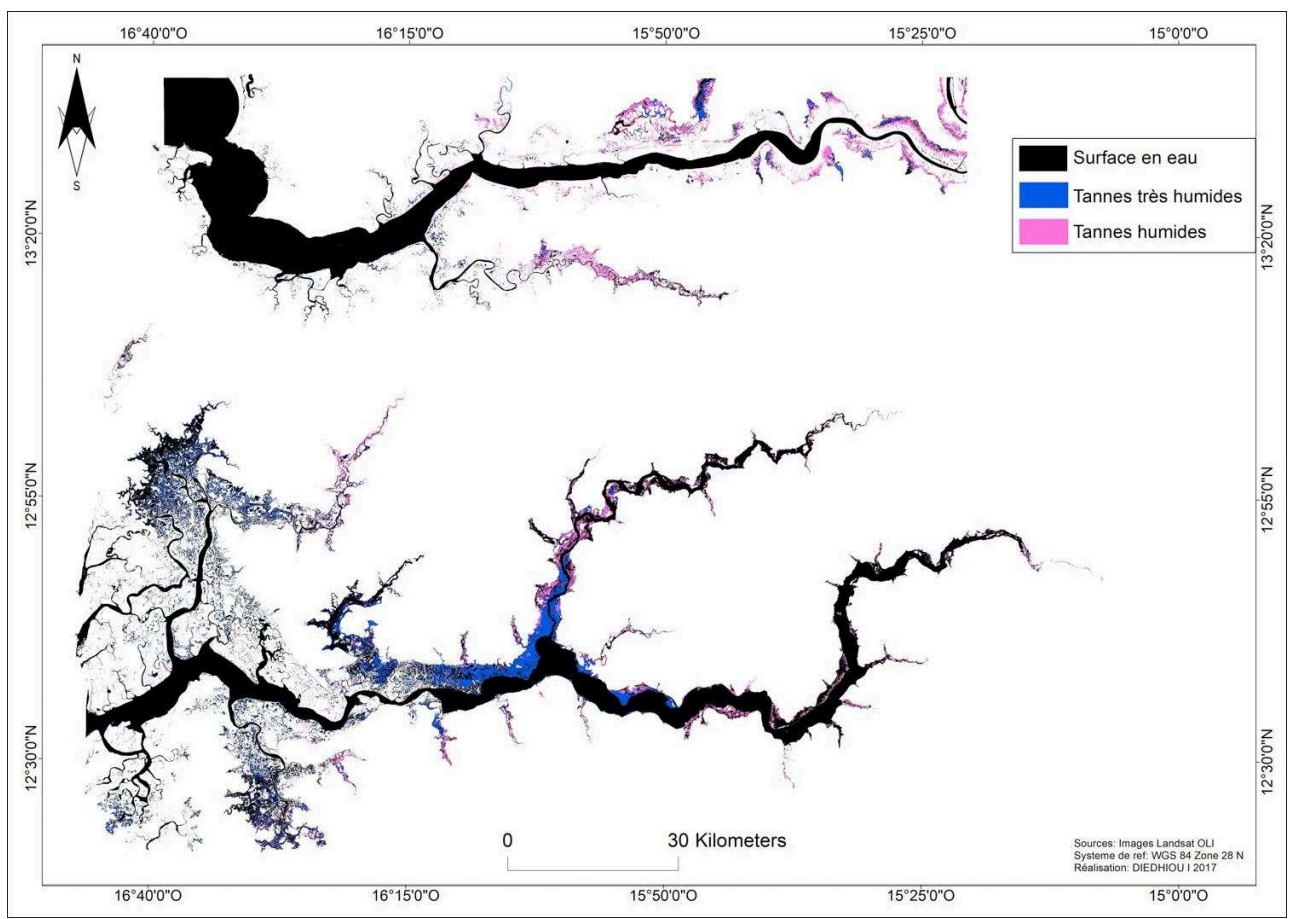

Les autres profils spectraux $(2,6,8,11$ et 12) correspondent à des surfaces qui ont la particularité d'avoir un pic plus ou moins accentué dans le proche infrarouge et une faible luminance dans le visible et dans le moyen infrarouge. Ce comportement spectral est caractéristique d'une végétation dense ; au sein des zones humides il ne peut s'agir ici que des boisements de mangrove. De la même manière que les tannes, les massifs de mangrove s'organisent suivant une toposéquence allant des zones de bas-fonds vers les hauts de pente. Généralement, les mangroves très denses (classes 6 et 11) occupent les parties les plus basses le long du littoral ou sur les rives des cours d'eau tandis que les mangroves ouvertes (classes 2, 8 et 12) couvrent les parties supérieures de la toposéquence (illustration 17). 
Illustration 17 - Typologie des boisements de mangrove

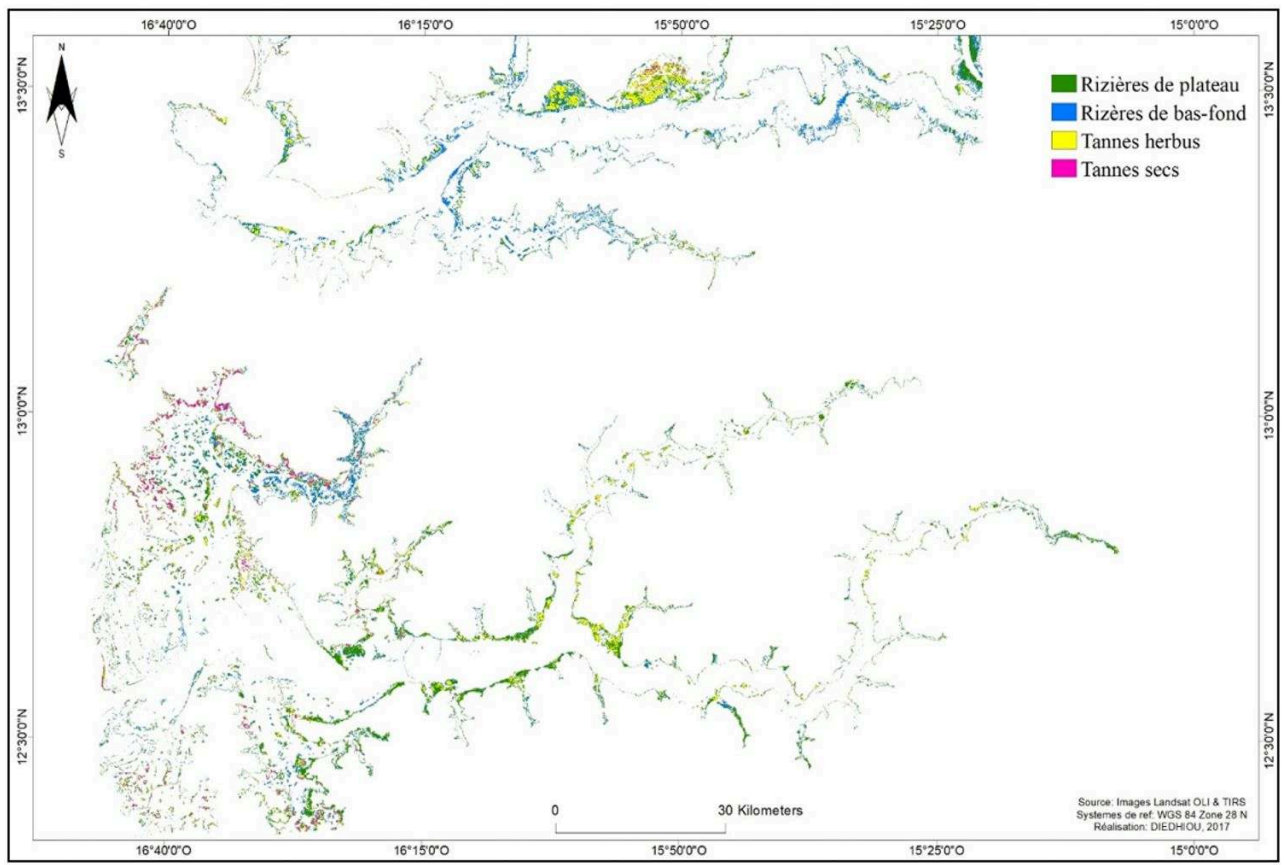

Les profils des classes 4 et 9 ne correspondent pas tout à fait aux signatures de référence générées pour les surfaces des zones humides présentées sur l'illustration 8 et tableau 4. Bien que caractérisés par un léger pic dans le proche infrarouge, on constate que la réponse de ces deux classes est plus importante dans le visible que celle de la mangrove ouverte mais moins élevée dans le moyen infrarouge. Cette caractéristique nous conduit à identifier ce type de surface comme étant des tannes recouverts d'une lame d'eau peu épaisse mais qui, contrairement aux classes 3 et 10 sont végétalisés. Cette interprétation est confortée par la relative similitude des profils de ces classes dans le visible avec celle des classes 3 et 13. Ces écarts subtils dans les comportements spectraux des différentes classes extraites illustrent à la fois la grande la variété et le continuum des paysages en milieu humide où l'on trouve à la fois des tannes humides nus et des tannes humides herbus. Ces deux catégories de comportements n'étant pas présents sur l'illustration 9, leur différenciation a été rendue possible par l'analyse des profils spectraux issus de la classification automatique en 15 classes. Dans ce cas, l'approche qui consiste à générer un grand nombre de profils spectraux s'est avérée utile puisqu'elle a permis de caractériser certaines surfaces, en comparant entre eux leur profils spectraux et au vu de leur localisation dans la toposéquence. Ainsi, le résultat de la classification au sein du milieu humide a mis en évidence un certain nombre de détails qui pourraient servir de base pour élaborer une cartographie détaillée de l'occupation du sol de la Sénégambie méridionale.

\section{Milieu de terre ferme}

La classification non supervisée des pixels de terre ferme (illustration 18) met en évidence quatre grandes catégories de profils spectraux (illustration 19). 
Illustration 18 - Classification non supervisée du milieu de terre ferme

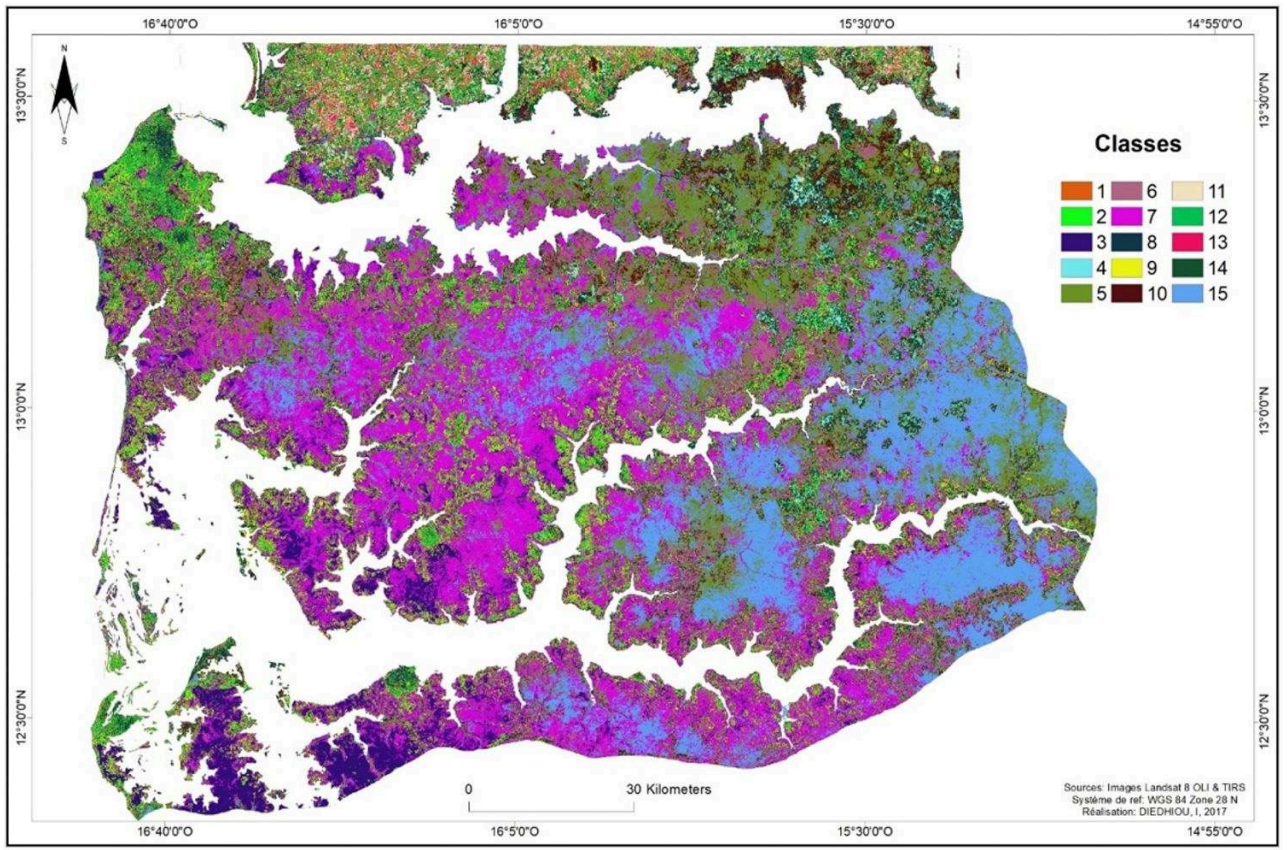

Un premier ensemble regroupe les classes $1,4,11$ et 13 dont les profils spectraux sont similaires au profil de référence des sols nus (illustration 8). Ces profils se démarquent des autres par une très forte luminance dans tous les canaux du visible à l'infrarouge moyen.

Illustration 19 - Profils spectraux des classes issues de la classification en milieu de terre ferme

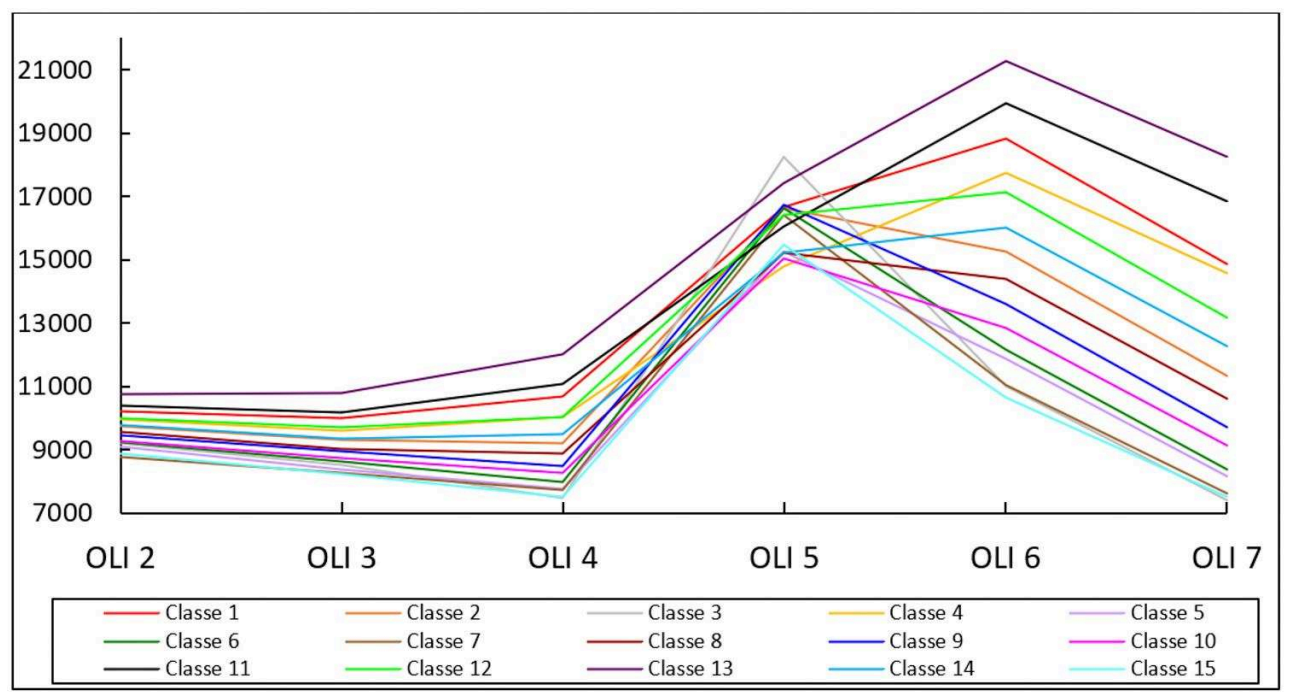

39 Au sein de cette catégorie de surfaces, on distingue d'une part, les sols secs très clairs (classe 13 et classe 11) dont la luminance croit graduellement dans les grandes longueurs d'ondes et d'autre part, les sols clairs (classe 1 et classe 4) dont la luminance est moins importante que celles des classes 13 et 11 (illustration 20a). Le deuxième ensemble est formé par les classes 2, 8, 12 et 14 dont les profils en forme de "toiture penchée vers l'avant " se caractérisent par deux pics d'importance variable respectivement dans le proche et le moyen infrarouge (illustration 20b). 
Illustration 20a - Profils spectraux des classes de terre ferme : sols nus

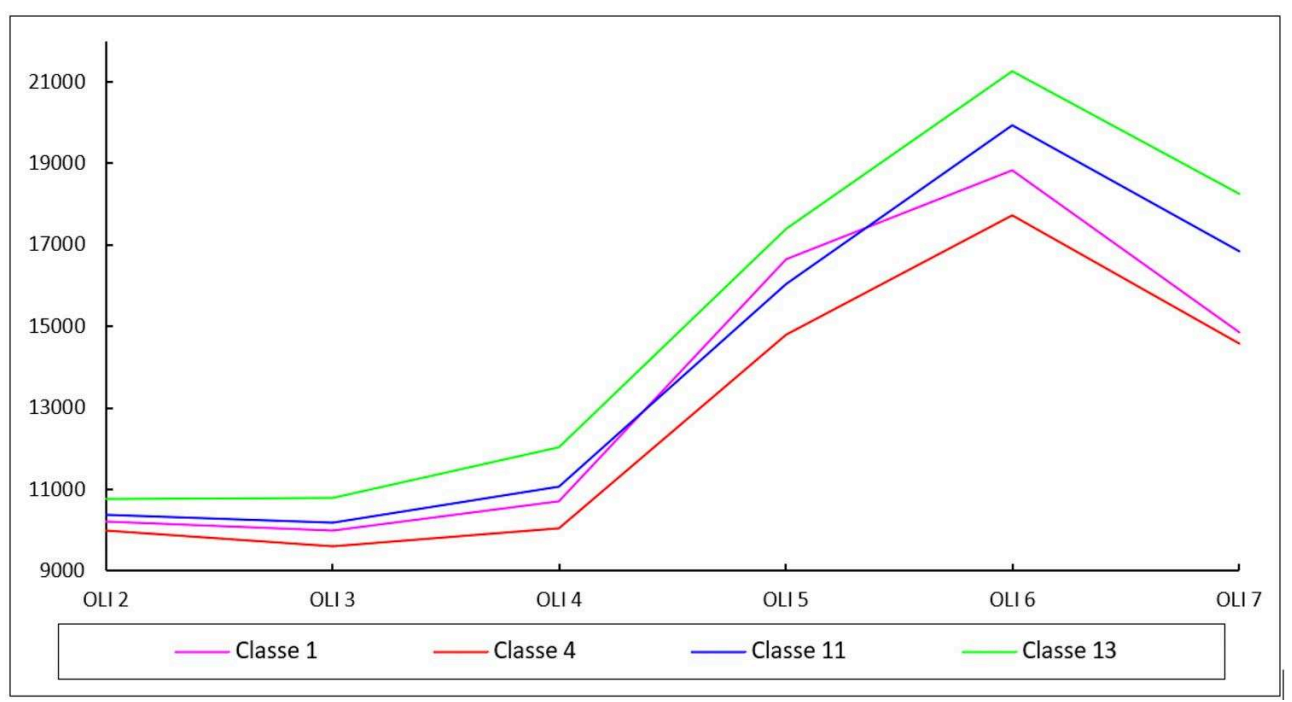

Illustration 20b - Profils spectraux des classes de terre ferme : zones de cultures

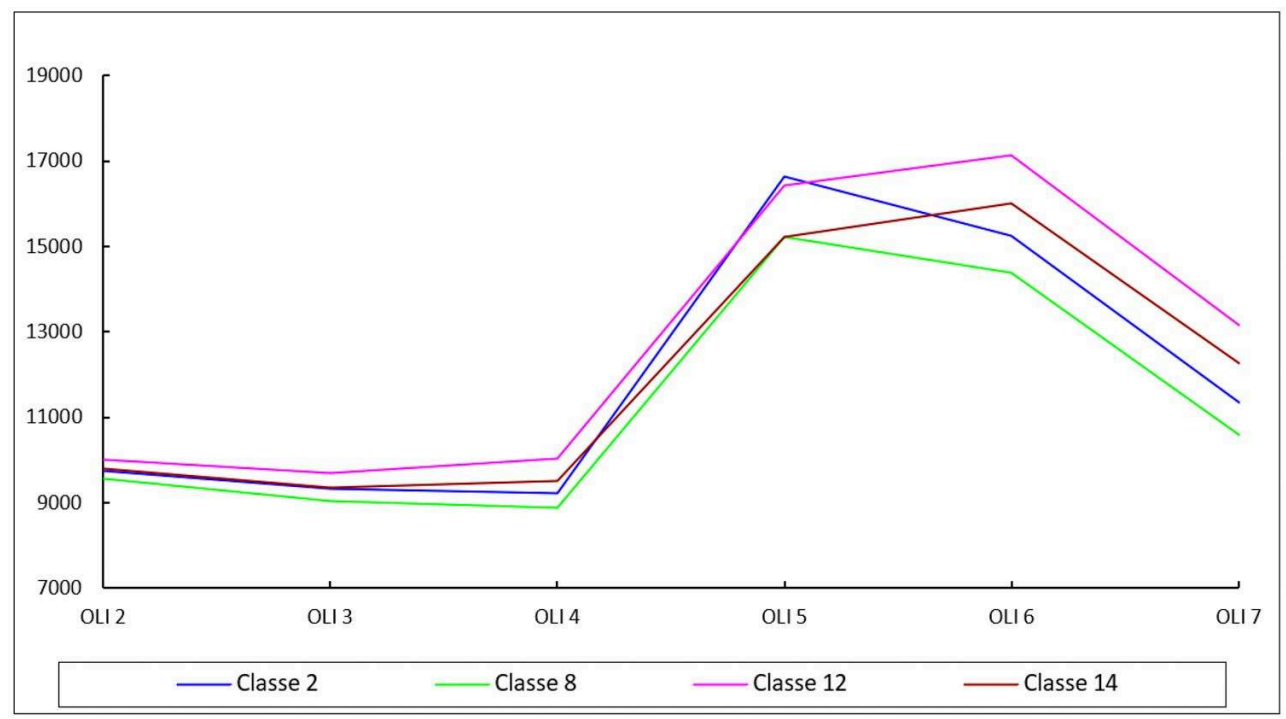


Illustration 20c - Profils spectraux des classes de terre ferme : boisements très ouverts

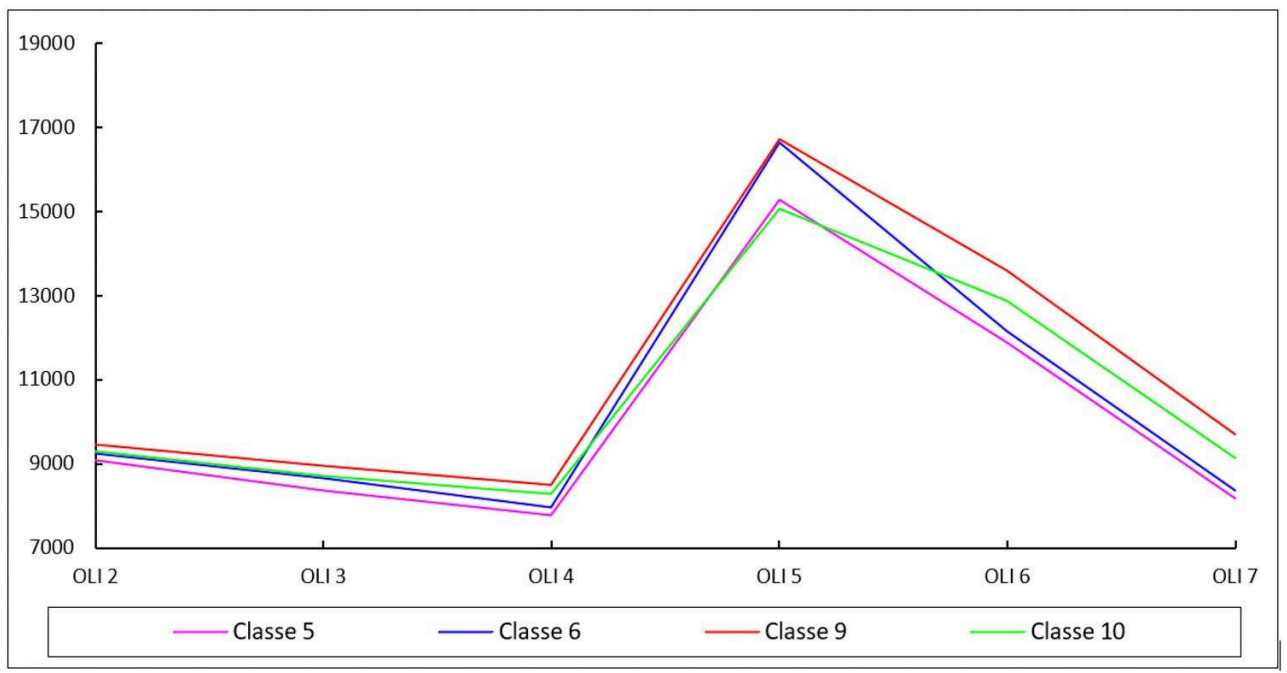

Illustration 20d - Profils spectraux des classes de terre ferme : boisements

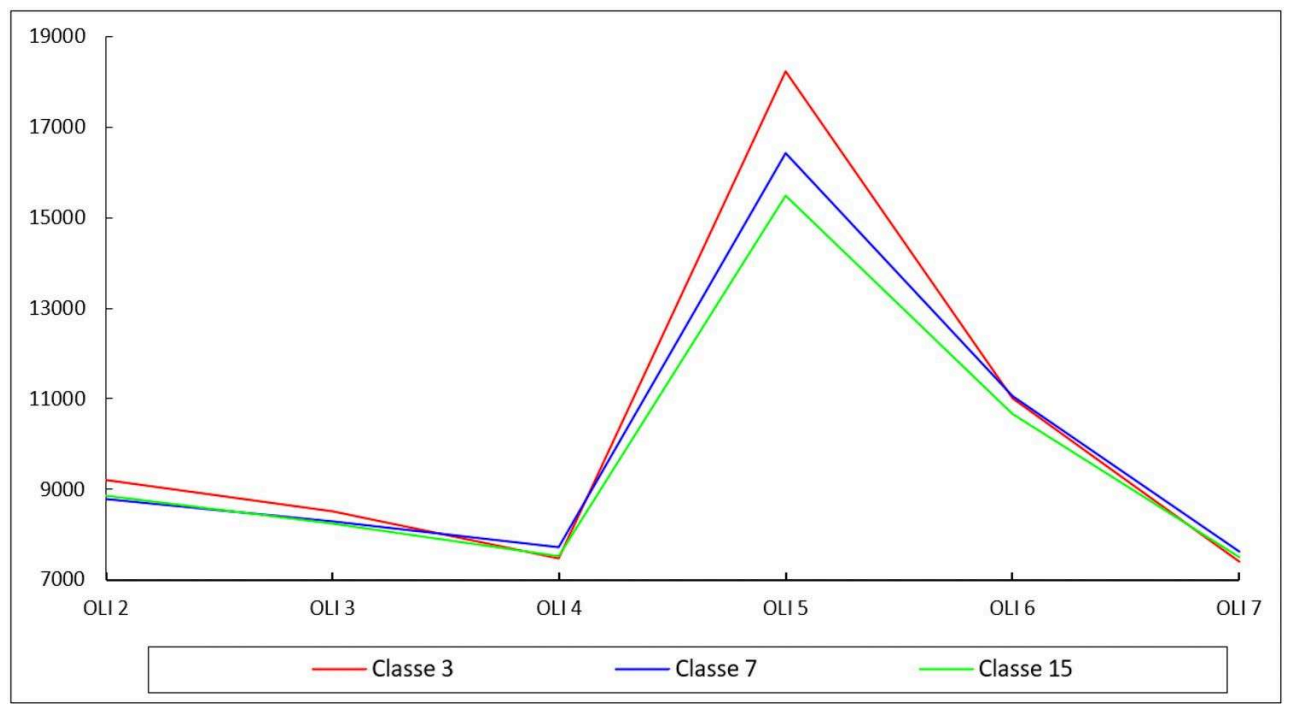

De manière générale, une telle configuration des profils traduit le comportement de surfaces composées d'une part, d'une végétation dont l'état phénologique ou la densité varie d'une catégorie à l'autre et d'autre part, de sols de teneur en eau et de teintes variables. En Sénégambie méridionale, ces surfaces composites correspondent à des espaces cultivés.

41 Le troisième ensemble regroupe les profils dont l'allure présente une pente douce entre le proche infrarouge et l'infrarouge moyen. Il s'agit ici des profils des classes 5, 6, 9 et 10, qui, contrairement à ceux qui ont été associés à des zones de cultures ont une luminance assez faible dans la partie visible du spectre électromagnétique (illustration 20c). Par ailleurs, les pics de luminance sont plus ou moins accentués pour les trois classes et leur réponse dans le moyen infrarouge varie également. Toutefois, si l'on le compare à celui des profils de référence des surfaces de terre ferme (illustration 8, tableau 3) le tracé général de ces profils indique qu'il s'agit de boisements très ouverts (illustration 21) 
Illustration 21 - Caractérisation des sols nus, des zones cultivées et des boisements très ouverts de terre ferme

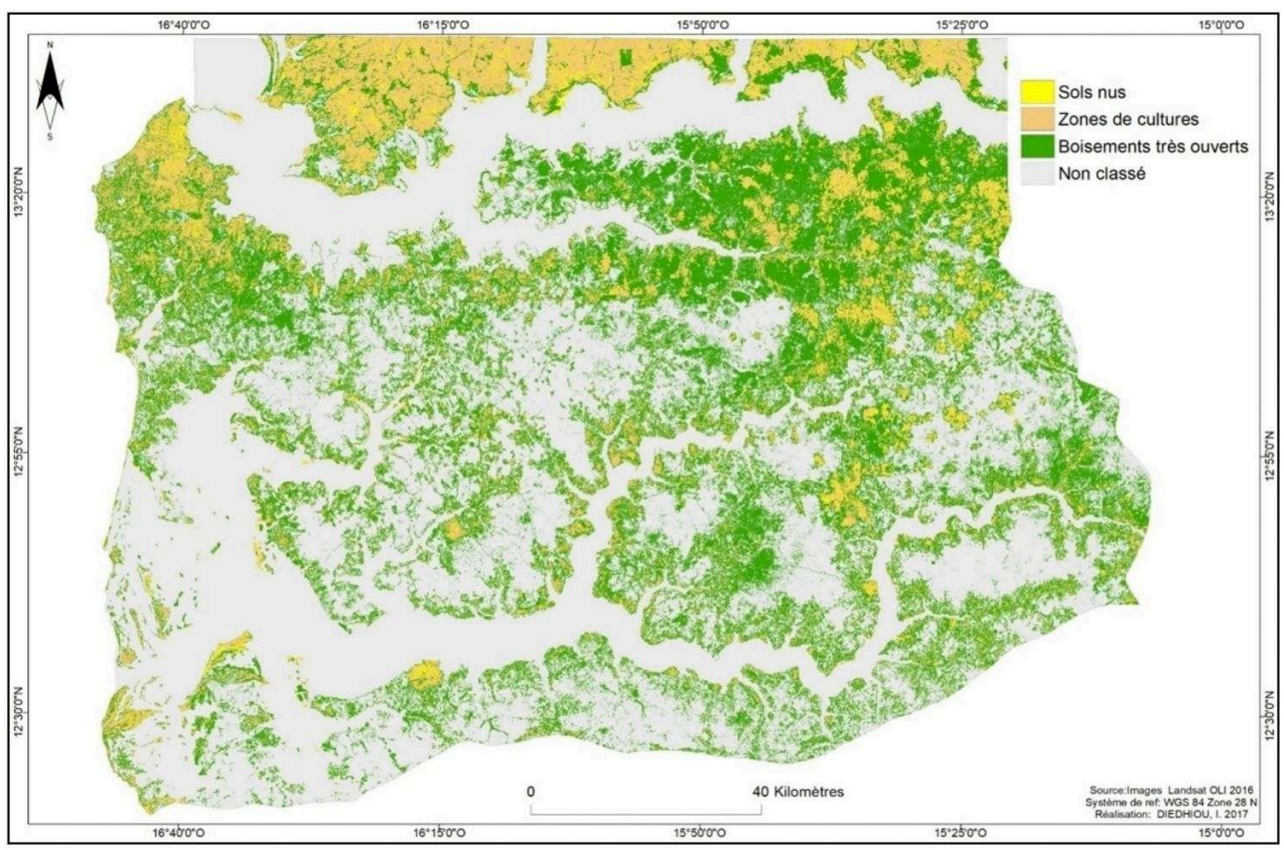

42 Le dernier ensemble comprenant les classes 3, 7 et 15, se singularise avant tout par la forme du profil spectral de ces classes qui se caractérise par une très forte luminance dans le proche infrarouge et une faible luminance dans les bandes du visible et du moyen infrarouge du spectre (illustration $20 \mathrm{~d}$ ). Comme le précèdent, cet ensemble illustre aussi les surfaces boisées qui, à la différence de celles mises en évidence sur l'illustration 20c, semblent plus fermées. Au-delà de cette apparente similarité du tracé, ces surfaces boisées fermées sont loin d'être homogènes en termes de densité. Dès lors, se pose la question de savoir dans quelle mesure peut-on considérer qu'un ensemble est homogène ? Un ensemble est dit homogène lorsque les profils spectraux présentent des similarités dans leur tracé comme c'est le cas des profils 5, 6 et 9, 10 sur l'illustration 20c. Dans ce cas de figure, l'ensemble est considéré comme tel et ne fait l'objet d'aucune subdivision

43 En revanche, si au moins l'une des courbes se distingue significativement des autres comme c'est le cas de la courbe 3 sur l'illustration 20d, on s'interroge sur le caractère significatif de cette distinction en termes d'occupation du sol. Avant de statuer définitivement sur l'homogénéité ou l'hétérogénéité de cet ensemble, nous avons eu recours à une Analyse en Composantes Principales (ACP) sur l'ensemble des bandes radiométriques afin d'accentuer les contrastes de luminance au sein de ce groupe de pixels à l'instar de ce qu'ont pratiqué, dans des cas similaires Andrieu et Mering (2008).

\section{Focus sur la classe « boisements denses »}

Le résultat de la classification à partir des néo-canaux issus de l'ACP du groupe de pixels dont les profils radiométriques correspondent au groupe D (illustration 20d) est présenté dans l'illustration 22. L'analyse des classes résultantes (illustration 23) est réalisée en se référant aux comportements spectraux des zones témoins de terre ferme (illustration 8, tableau 3). 
Illustration 22 - Classification non supervisée de la classe « boisements denses de terre ferme » à partir des résultats de l'ACP

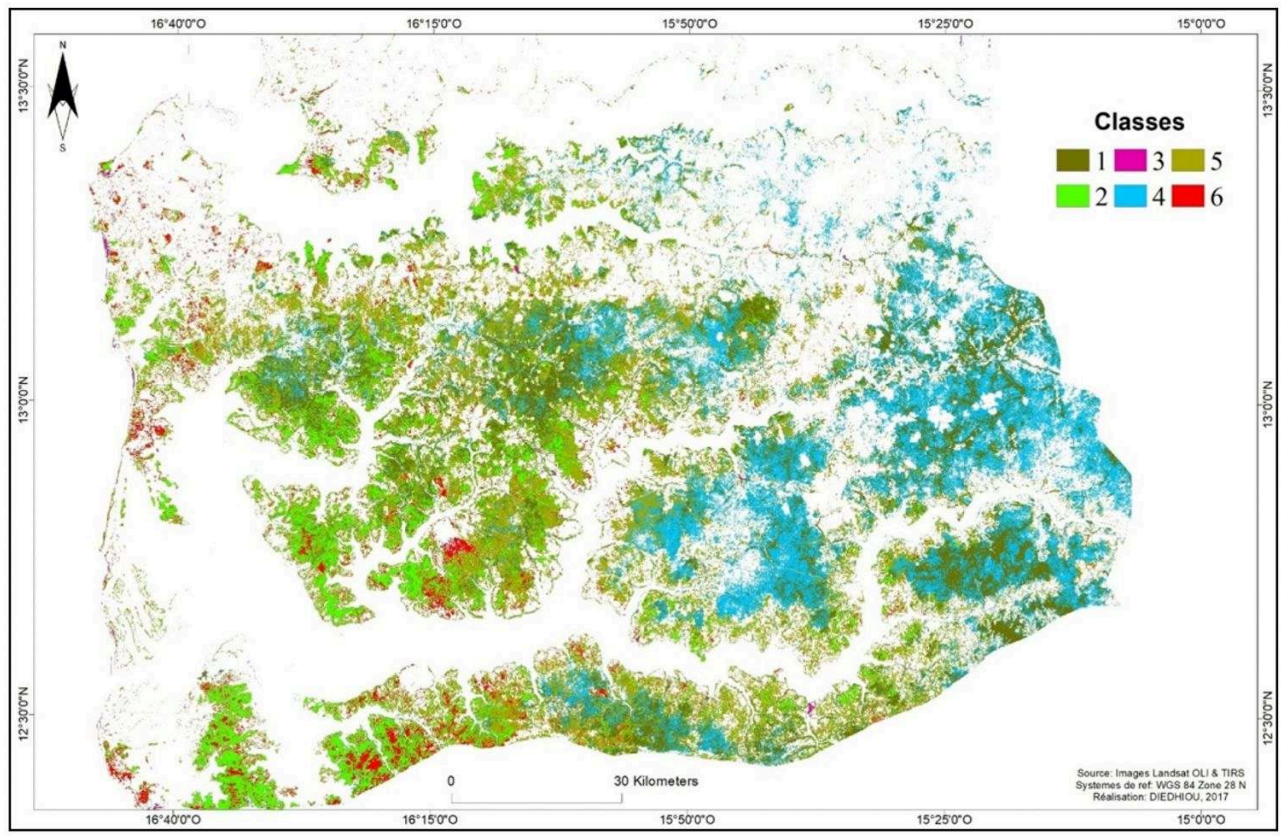

Illustration 23 - Profils spectraux de la classification de la végétation à partir des résultats de l'ACP

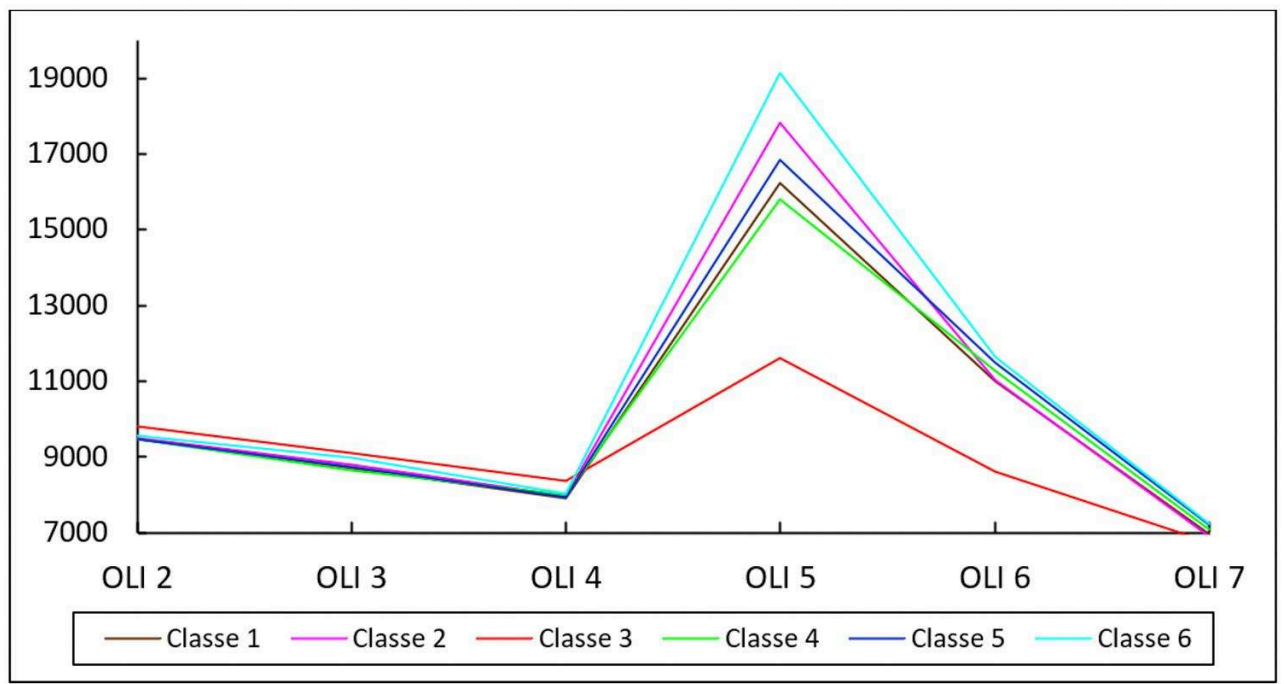

La première catégorie est constituée par les classes $1,2,4,5$ et 6 . Cet ensemble de classes correspond aux boisements de terre ferme qui se caractérise par une faible luminance dans la partie visible du spectre, un pic élevé dans le proche infrarouge suivi d'une légère diminution de la luminance dans l'infrarouge moyen. Selon les valeurs de luminance, différentes densités de peuplements se distinguent à l'intérieur de cet ensemble. Il s'agit :

D'une part, les surfaces boisées denses qui se caractérisent par une luminance très faible dans le visible et un pic très important dans le proche infrarouge (classe 2 et classe 6). Selon l'importance de la luminance, on peut subdiviser cette classe en deux sous-types: l'ensemble des boisements denses représenté par la classe 2 et les groupements d'arbres à très forte activité photosynthétique (classe 6). 
D'autre part, des boisements qui se singularisent par une luminance plus faible dans le proche infrarouge du spectre électromagnétique que celle des boisements denses Cette diminution sensible de la luminance peut être due à la présence de traces de brûlis. Ce type de surfaces boisées est ici interprété comme étant des boisements ouverts (illustration 24).

La dernière catégorie représentée par la classe 3 ne peut en aucun cas être associée à des boisements de terre ferme: en effet, l'analyse du profil spectral des surfaces associées montre qu'elles présentent à la fois une activité chlorophyllienne beaucoup plus faible et une teneur en eau beaucoup plus importante que le couvert végétal de terre ferme. En se référant aux signatures spectrales des zones témoins en milieu humide(illustration 9, tableau 4), on peut supposer qu'il s'agit des tannes herbus. On constate, à travers cet exemple, que la segmentation des scènes en deux types de milieu n'a pas été totalement réussie, puisque l'on a pu détecter des composantes du milieu humide, ici les tannes, au sein de ce qui est censé constituer le milieu de terre ferme. Cependant dans la mesure où ces surfaces, occupent une très faible part de de la scène, on peut considérer que l'approche de segmentation adoptée en amont est globalement valide. De plus, dans ce cas, le recours à l'ACP a permis de dététecter une hétérogénéité de comportement spectral au sein du groupe de pixels considéré qui avait été négligée lors de la première étape de classification.

Illustration 24 - Résultat de la classification non supervisée de la classe boisements à partir des résultats de l'ACP

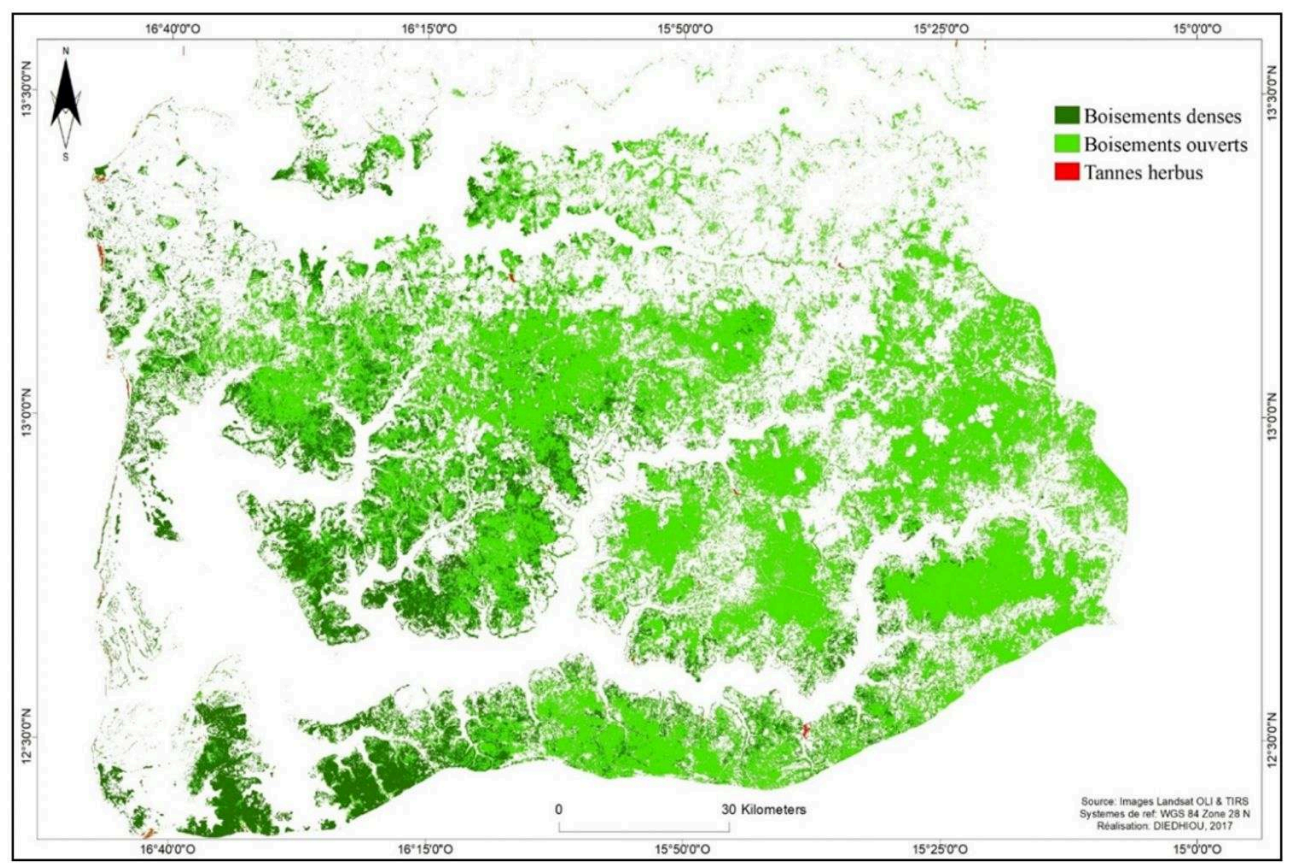




\section{Cartographie de l'occupation du sol et de ses changements}

\section{Cartographie de l'occupation du sol}

A cette étape, nous avons fusionné les différentes classes crées pour chacun des milieux dans le but de ne représenter que les taxons définis en amont (tableau 2). Ainsi les classes mangroves très denses, denses, ouvertes et très ouvertes ont été regroupées entre elles pour ne former qu'une seule classe thématique : celle des boisements de mangrove. Il en est de même pour les tannes et/ou rizières. Concernant les milieux de terre ferme, certaines classes à fort effectif ont fait l'objet d'une subdivision, ce qui a permis de représenter l'ensemble des taxons pour ce type de milieu conformément à ce qui avait été prévu initialement (tableau 2). Pour produire la carte d'occupation des sols de l'ensemble de la région d'étude (illustration 25), les cartes d'occupation du sol de chaque type de milieu ont été additionnées.

Illustration 25 - Cartographie de l'occupation du sol de la Sénégambie méridionale en 2016

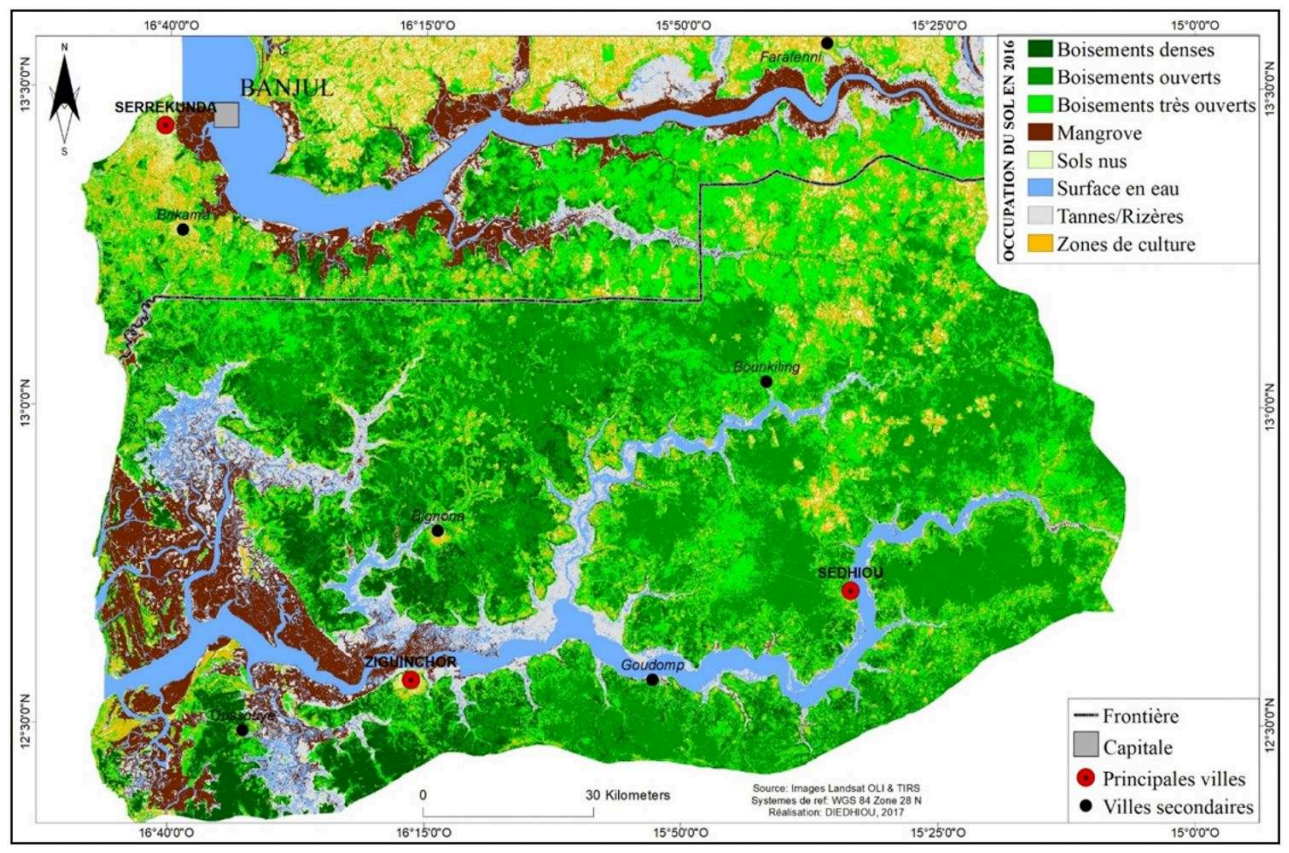

\section{Évaluation de la qualité de la carte de 2016}

La longue séquence d'opérations détaillée dans ce qui précède à travers l'exemple du traitement de la scène Landsat OLI de 2016 a pour résultat une carte de l'occupation du sol en 2016 (illustration 25). Afin d'évaluer la qualité la carte de 2016, et en supposant que l'occupation du sol a peu évolué depuis 2014, date à laquelle nous disposons de zones témoins correspondant à échantillons de réalités terrain en Basse Casamance (illustration 7), une matrice de confusion a été calculée à partir de ces zones témoins (tableau 6).

Comme on peut le constater sur ce tableau, pour chaque classe, les erreurs de commission et d'omission sont négligeables. Quant au coefficient kappa, il est 
suffisamment proche de 1 pour que l'on puisse en conclure que la carte de 2016 satisfait aux exigences classiquement requises en Télédétection quant à la qualité de la carte.

Tableau 6 - Matrice de confusion relative à la carte d'occupation du sol de la Sénégambie méridionale de 2016 basée sur la donnée des zones témoins issues de la carte de 2014 sur la Basse Casamance

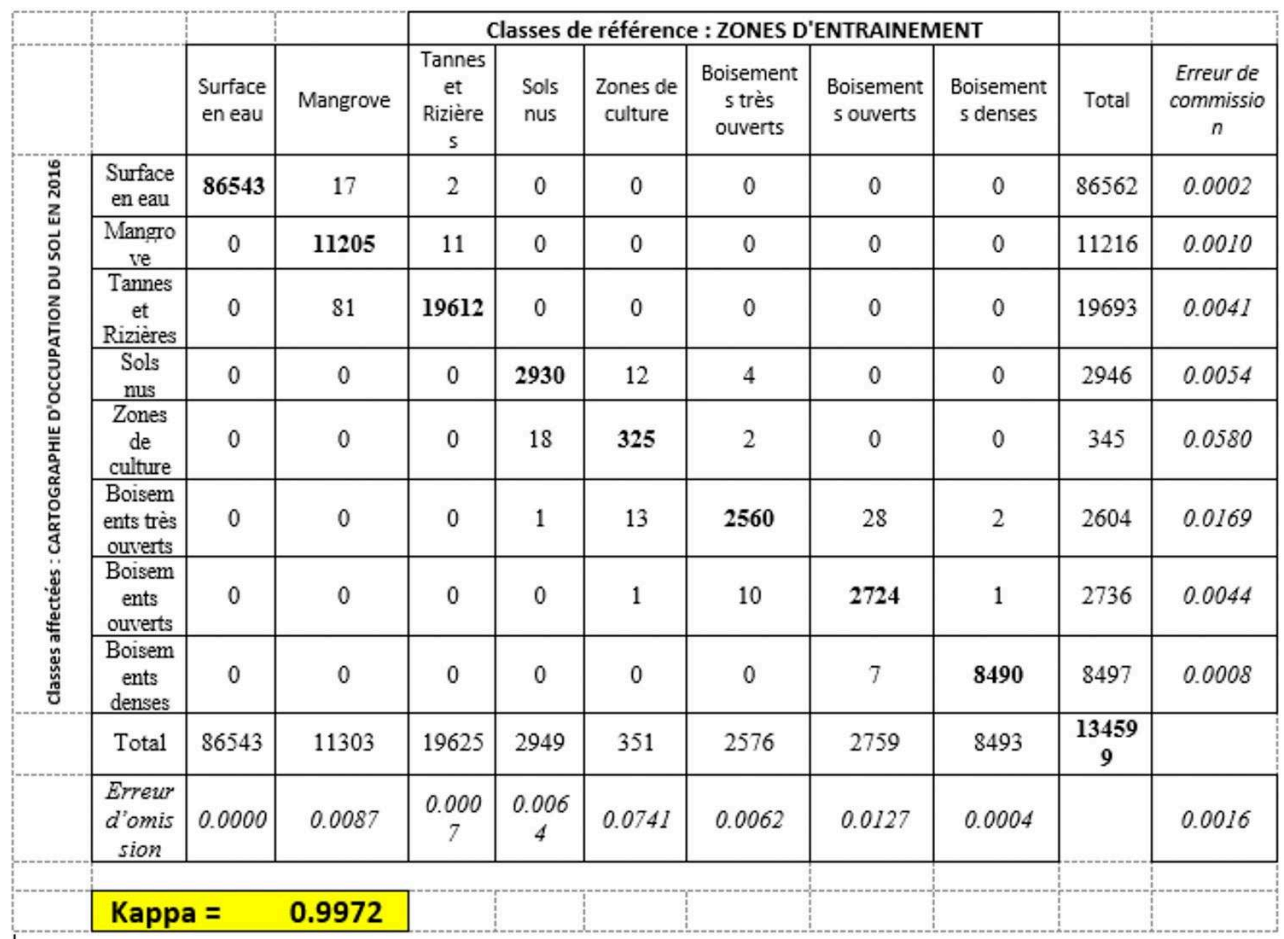

\section{Cartographie des changements au cours de la période (1972-2016)}

$\mathrm{Au}$ lieu de réaliser plusieurs cartes diachroniques des changements à deux dates, nous avons choisi de représenter sur une seule carte la séquence des changements survenus à chacune des périodes comprises entre les quatre dates $(1972,1988,2000,2016)$. Pour atteindre cet objectif, la procédure numérique appliquée aux cartes correspondant aux quatre dates a été la suivante :

51 On multiplie la première carte d'occupation du sol (1972) par 1000, puis on l'additionne avec la carte de la deuxième date (1988) qui est multipliée par 100 puis additionnée avec la carte de la troisième date (2000), qui est multipliée par 10 avant d'être additionnée avec la carte de la dernière date (2016). La procédure est résumée par la formule suivante:

Carte d'occupation du sol de $1972 * 1000+$ Carte d'occupation du sol de $1988 * 100+$

Carte d'occupation du sol de $2000 * 10+$ Carte d'occupation du sol de 2016 .

En fonction du nombre de classes qui compose le type de milieu, la carte résultante de cette opération présente des valeurs de pixels allant de 1111 (première classe) à N*1000 (dernière classe). Par exemple pour le cas du milieu humide qui est subdivisé en trois classes d'occupation du sol (tableau 6), les pixels présentent des valeurs qui seront des combinaisons des codes de 1 à 3 sur 4 positions.

53 Ce type d'opération est effectué pour toutes les classes du milieu humide et les valeurs de pixels sont interprétées suivant la nomenclature définie en amont (tableau 7). 
Tableau 7 - Typologie des changements d'occupation du sol

\begin{tabular}{|c|c|c|c|c|}
\hline & \multicolumn{2}{|c|}{ Milieu humide } & \multicolumn{2}{|l|}{ Terre ferme } \\
\hline \multirow{2}{*}{ Stabilités } & Mangrove & Tannes et rizières & Boisements & Zones de Cultures \\
\hline & \multicolumn{2}{|c|}{ Surfaces en eau } & \multicolumn{2}{|l|}{ Sols nus } \\
\hline \multirow{4}{*}{ Progressions } & \multicolumn{2}{|l|}{ Anciennes } & \multicolumn{2}{|l|}{ Anciennes } \\
\hline & \multicolumn{2}{|c|}{ Temporaires } & \multicolumn{2}{|l|}{ Temporaires } \\
\hline & \multicolumn{2}{|l|}{ Récentes } & \multicolumn{2}{|l|}{ Récentes } \\
\hline & \multicolumn{2}{|l|}{ Continues } & \multicolumn{2}{|l|}{ Continues } \\
\hline \multirow{4}{*}{ Régressions } & \multicolumn{2}{|l|}{ Anciennes } & \multicolumn{2}{|l|}{ Anciennes } \\
\hline & \multicolumn{2}{|c|}{ Temporaires } & \multicolumn{2}{|l|}{ Temporaires } \\
\hline & \multicolumn{2}{|l|}{ Récentes } & \multicolumn{2}{|l|}{ Récentes } \\
\hline & \multicolumn{2}{|l|}{ Continues } & \multicolumn{2}{|l|}{ Continues } \\
\hline & & & Brulis & Nuages \\
\hline
\end{tabular}

\section{Interprétation des changements des sols}

Les codes résultant de la procédure définie ci-dessus sont interprétés comme suit : les chiffres des milliers représentent la classe en 1972, celui des centaines, ce qu'elle est devenue en 1988, celui des dizaines décrivent son état en 2000 et celui des unités, la situation en 2016. C'est ainsi que des valeurs comme 1111, 2222 et 3333 représentent les surfaces du milieu humide (les surfaces en eaux, la mangrove, les tannes et rizières) n'ayant pas connu de changements durant toute la période étudiée. De façon analogue, pour la terre ferme des codes suivants: 4444, 5555, 6666, 7777 et 8888 correspondent respectivement à la stabilité des classes : sols nus, zones de culture, boisements très ouverts, boisements ouverts et boisements denses ${ }^{12}$.

En milieu humide, il peut arriver que des pixels appartenant à la classe d'occupation du sol comme les "tannes et/ou rizières" se transforment en surfaces en eau, ou à l'inverse que ces dernières deviennent tannes et/ou rizières. Ces types de transformation ne sont pas rares dans ce type de milieu dans la mesure où les surfaces couvertes par l'eau à un instant donné peuvent évoluer en fonction des marées ou d'autres phénomènes météorologiques (tempêtes) ou hydrodynamiques. Mais, compte tenu de notre objectif qui est d'étudier la dynamique des paysages forestiers, nous négligerons de tels changements pour ne retenir que les changements correspondant 
au passage entre de mangrove et absence de mangrove (tannes et rizières, eau) ou à la transformation inverse. Ainsi dans le cas où les tannes et/ou rizières voire des surfaces couvertes d'eau peu profonde se transforment en mangrove on parlera de la progression de la mangrove ; à l'inverse lorsque la mangrove se transforme en tannes et/ou rizières ou en surface couverte d'eau on parlera de régression de la mangrove. Selon la période, ce type de changement peut être ancien (entre 1972 et 1988 exclusivement), temporaire (entre la deuxième et troisième date exclusivement), récent (entre les deux dernières dates) ou encore continu (évolution qui se poursuit tout au long de la période étudiée).

L'interprétation se faisant de la gauche vers la droite comme décrit plus haut, les valeurs de pixels 1211 et 1123 peuvent être interprétées comme étant des progressions anciennes et temporaires de la mangrove sur les surfaces recouvertes d'eau ${ }^{13}$. À titre d'exemple, dans le cas du premier code (1211), les transformations (de surfaces recouvertes d'eau en surfaces occupées par la mangrove) se sont produites uniquement en 1988, ce qui n'est pas le cas pour le deuxième code (1123) qui représente des changements qui sont intervenus en 2000 après une période de stabilité (entre 1972 et 1988). On pourrait ainsi résumer la signification de du code 1123: «les surfaces recouvertes d'eau entre 1972 et 1988, se sont transformées en mangrove en 2000, puis en tannes et/ou rizières en 2016 ». De la même manière, on peut aussi interpréter la valeur de pixels 3312 comme une progression récente et 3222 comme une progression continue de la mangrove. La progression récente illustre la dynamique actuelle ou en cours dans les zones humides (entre 2000 et 2016), après une longue période de stabilité des tannes et/ou rizières (entre 1972 et 2000). La dynamique de progression continue concerne les surfaces qui, après avoir subi des transformations de tannes et rizières en mangrove au début de la période d'étude sont restées par stables par la suite. Le code (3222) représente ce type de dynamique.

57 De façon analogue, les codes sont interprétés en termes de dynamiques de régression de mangrove qui peuvent se faire aussi bien au profit des tannes et/ou rizières (2223 et 2322) qu'au profit des surfaces recouvertes d'eau (2212 et 2111). Comme pour les progressions, les valeurs de pixels déterminent la temporalité de ces régressions. Ainsi les codes 2223 et 2322 sont respectivement être interprétés comme décrivant des dynamiques de régression récente et ancienne et les codes (2212 et 2111) comme des régressions temporaires (resp. continues).

58 La même approche a été utilisée pour cartographier les dynamiques des couvertures forestières de terre ferme. Une fois que les changements au sein des deux types de milieu ont été clairement identifiés, une carte des changements en termes d'évolution (progression ou régression) du couvert forestier au sein des deux catégories de milieu physique a été réalisée (illustration 26). 
Illustration 26 - Cartographie des changements d'occupation du sol en Sénégambie méridionale entre 1972 et 2016

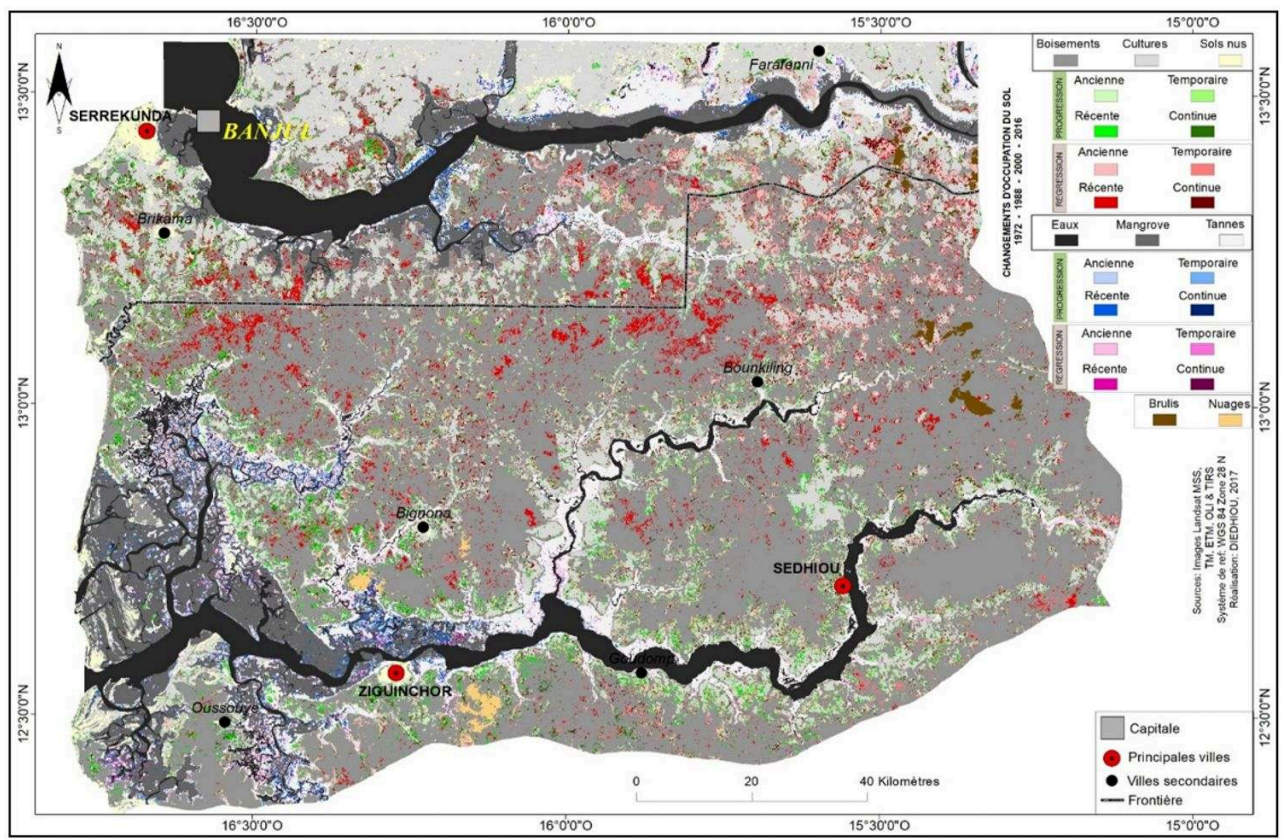

\section{Évolution des paysages forestiers durant la période d'étude (1972-2016)}

L'analyse de l'illustration 26 montre une dynamique très contrastée entre le nord et le sud de la région d'une part, mais aussi entre l'ouest et l'est où, à mesure que l'on s'éloigne du littoral on passe progressivement d'un milieu humide à celui de terre ferme.

Tableau 8 - Quantification (en hectares) de l'évolution des paysages forestiers sénégambiens de 1972 à 2016

\begin{tabular}{|l|l|l|l|l|}
\hline & Terre ferme & Hectares & Zones humides & Hectares \\
\hline \multirow{4}{*}{ Stabilité } & Boisements & 1056987 & Eaux & 184585 \\
\cline { 2 - 6 } & Zones de culture & 286755 & Mangrove & 138681 \\
\cline { 2 - 6 } & Sols nus & 60608 & Tannes & 177050 \\
\hline \multirow{5}{*}{ Progression du couvert forestier } & Ancienne & 8712 & Ancienne & 6515 \\
\cline { 2 - 6 } & Temporaire & 7385 & Temporaire & 1949 \\
\cline { 2 - 6 } & Récente & 13392 & Récente & 9367 \\
\cline { 2 - 6 } & Continue & 6884 & Continue & 1290 \\
\hline \multirow{2}{*}{ Régression du couvert forestier } & Ancienne & 10916 & Ancienne & 7113 \\
\cline { 2 - 6 } & & &
\end{tabular}




\begin{tabular}{|l|l|l|l|l|}
\cline { 2 - 5 } & Temporaire & 12575 & Temporaire & 8796 \\
\cline { 2 - 6 } & Récente & 39353 & Récente & 2188 \\
\cline { 2 - 5 } & Continue & 18738 & Continue & 3221 \\
\hline & Brulis & 7425 & Nuages & 4387 \\
\hline
\end{tabular}

\section{Évolution des paysages forestiers de terre ferme}

60 La partie nord se démarque du reste de la région par l'importance des régressions récentes du couvert forestier (illustration 26). Ce type de dynamique (en rouge vif sur la carte de cette illustration) qui s'observe tout particulièrement le long de la frontière sénégalo-gambienne, illustre l'ampleur du phénomène de déboisement lié au trafic de bois vers la Gambie voisine (Treanor, 2015 ; Diedhiou et Mering, 2019). En effet, la perméabilité des frontières consécutive à l'insécurité qui persiste dans ce secteur a favorisé l'essor d'un trafic illicite de produits ligneux (Marut, 2013).

$61 \mathrm{Au}$ nord-est, on observe des régressions anciennes (en rose sur la carte de l'illustration 26), caractéristiques des anciens fronts de défrichement pour la mise en culture tandis que celles continues (en marron sur la carte de l'illustration 26) localisées sous forme de semis de taches au centre de la région peuvent témoigner d'activités traditionnelles de prélèvement de bois pour les usages domestiques.

Les progressions récentes du couvert ligneux (en vert vif sur la carte de l'illustration 26) prédominent quant à elles le long de la façade atlantique et de part et d'autre des rives de la Casamance. Ce type de dynamique semble être le résultat d'une évolution des pratiques rurales avec la reconversion des anciens espaces agricoles en zones sylvicoles (anacardiers et vergers) davantage qu'une véritable régénération naturelle de la végétation ligneuse. En effet la variabilité pluviométrique a renforcé l'impact économique des cultures fruitières de rente comme celle de l'anacardier (Samb et al., 2018) dans le revenu des ménages ${ }^{14 .}$

63 Le sud de la région, quant à lui, reste dominé par les progressions des boisements qui, selon les zones peuvent être anciennes (en vert clair), continues (en vert sombre) ou récentes. Plusieurs facteurs expliquent ce type de dynamique : outre le développement des plantations de vergers et d'anacardiers (Ndiaye, 2017) il peut s'agir d'une la régénération naturelle de la végétation dont la principale cause reste l'instabilité persistante de la zone frontalière au sud de la Casamance ${ }^{15}$ (Robin, 2008) qui a entrainé un certain abandon des activités rurales et donc un enfrichement des espaces anciennement cultivés. Dans un tel contexte de déprise rurale, le faible taux de prélèvement de ligneux, pourrait expliquer les progressions du couvert végétal observées au cœur des boisements dans certaines zones de cette partie de la Casamance.

64 Le bilan global de la dynamique des paysages forestiers sur terre ferme (illustration 27) est celui d'un recul important du couvert ligneux, qui, s'il ne date pas d'hier, a pris de l'ampleur depuis les années 2000, en particulier dans la partie septentrionale de la région d'étude, le long de la frontière entre la Gambie et le Sénégal. 


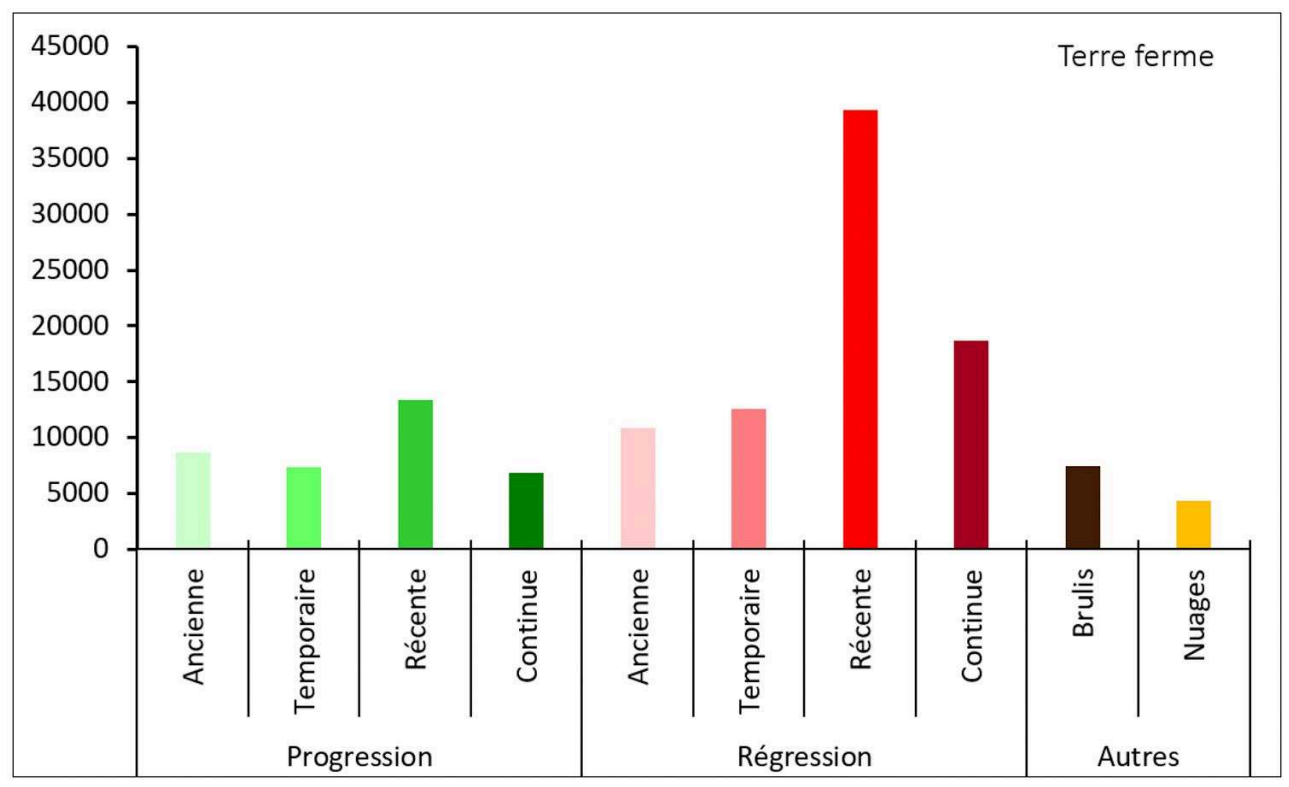

\section{Évolution des paysages en milieu humide}

Contrairement à ce qui s'est passé sur terre ferme, le bilan global de l'évolution des paysages en milieu humide est celui de la progression récente de la mangrove. En effet, si le déficit pluviométrique des années 70 et 80 a été à l'origine d'une régression importante de la mangrove (en violet sur la carte illustration 26 et sur la illustration 28), et si les traditionnels prélèvements de bois de palétuviers pour différents usages locaux (construction de pirogues, de fours pour fumer le poisson,....) qui se sont poursuivis jusqu'à une période récente (Bassène, 2016) ont causé un recul de la mangrove au voisinage des lieux densément peuplés (en rose fuchsia et violet foncé sur la carte illustration 26 et sur l'illustration 28), c'est bien la progression actuelle de la mangrove (en bleu vif sur la carte de l'illustration 26 et sur l'illustration 28) qui constitue le fait marquant de l'évolution des paysages en milieu humide durant la période étudiée. Cette dynamique illustre la capacité de résilience des écosystèmes de mangrove qui ont réussi à se régénérer lorsque les conditions pluviométriques sont redevenues favorables : la lixiviation au sel et au soufre du sol a permis à la mangrove de repousser avec parfois de nouveaux semis de Rhizophora mangle et des ventouses d'arbres et d'arbustes presque morts d'Avicennia africana (Andrieu 2018, Soumaré et al., 2020). À cette régénération naturelle s'ajoute le reboisement récent par les populations avec ou sans l'appui d'ONG (Bassène et al., 2013). 
Illustration 28 - Dynamique d'évolution des paysages en milieu humide

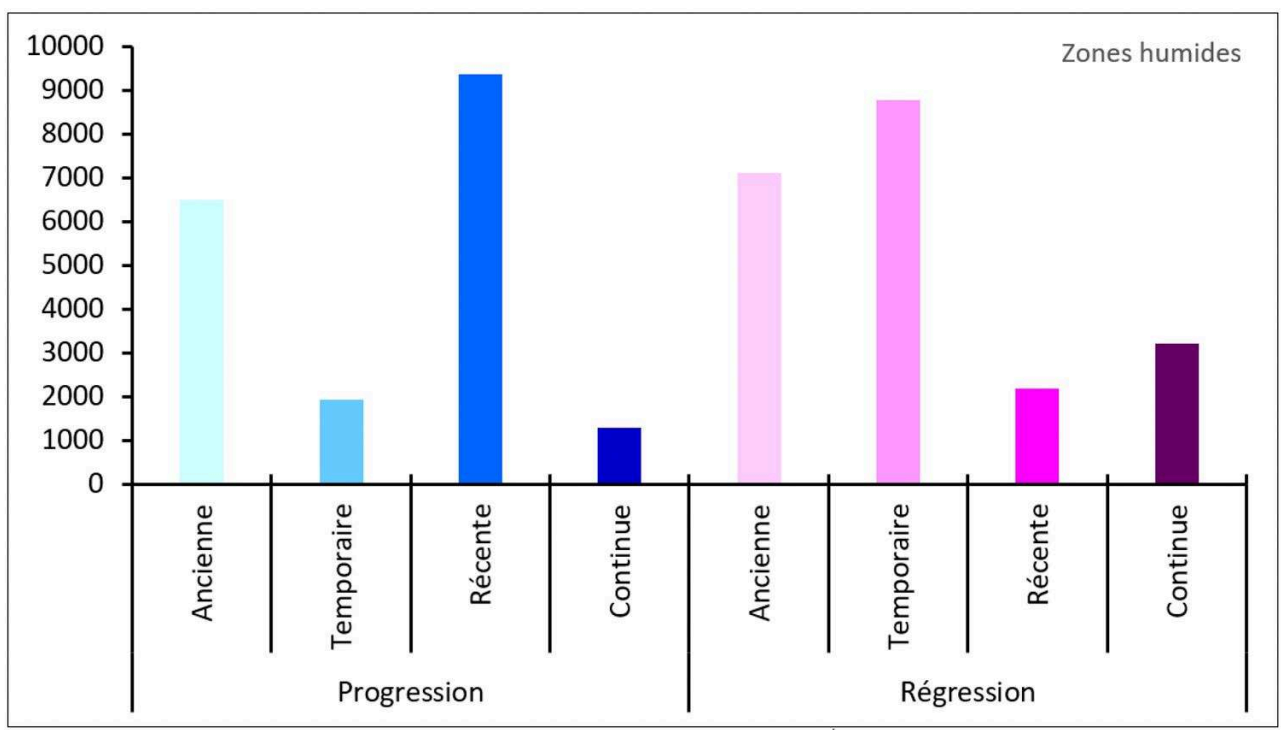

\section{Conclusion}

Si la cartographie de l'occupation du sol et de ses changements en Sénégambie méridionale a été réalisée à l'aide d'une méthode classique de classification multispectrale non supervisée, l'interprétation des profils spectraux des classes résultantes a pu bénéficier de la connaissance du comportement spectral de zones témoins extraites d'une carte récente d'occupation du sol d'une partie de la région d'étude.

Le travail de comparaison à l'origine de l'identification des classes par le biais de leurs comportements spectraux a permis dans un premier temps de séparer le milieu humide de celui de terre ferme, ce qui a considérablement réduit le risque de confusion entre classes d'occupation du sol spectralement proches bien qu'appartenant à deux milieux différents. La discrétisation des différents types de couverts ligneux de terre ferme selon leur degré de densité, indispensable pour analyser de l'évolution des paysages forestiers, a également été grandement facilitée par une telle approche. Enfin, la donnée de zones témoins des différentes catégories d'occupation du sol a permis d'évaluer la qualité de la carte correspondant à la date la plus récente.

La cartographie de l'évolution de l'occupation du sol durant la période étudiée a confirmé les résultats déjà connus selon lesquels l'amélioration des conditions pluviométriques depuis la fin des années 90 a bénéficié aux formations de mangrove. Par contre, très loin d'un constat d'une progression globale du couvert ligneux sur terre ferme, c'est l'importance des déboisements le long de la frontière entre le Gambie et le Sénégal durant la période récente qu'a mis en évidence la carte des changements. La reproduction de la démarche décrite ici pourrait permettre à l'avenir d'alimenter une base de données relative aux comportements spectraux des principales formations végétales de la région et de participer ainsi à un suivi régulier des paysages Sénégambiens. 


\section{BIBLIOGRAPHIE}

Alexandre F., Mering C., 2019. Perception et représentation des changements socioenvironnementaux dans les sociétés rurales en Afrique de l'Ouest sahélienne et soudanienne, L'Espace géographique, vol. tome 48, $\mathrm{n}^{\circ}$. 2, pp. 97-102.

Andrieu J., 2018. Land cover changes on the West-African coastline from the Saloum Delta (Senegal) to Rio Geba (Guinea-Bissau) between 1979 and 2015. European Journal of RemoteSensing, vol. $51, \mathrm{n}^{\circ}$ 1. p. $314-325$

Andrieu J., Mering C., 2008. Cartographie par télédétection des changements de la couverture végétale sur la bande littorale ouest-africaine : exemple des Rivières du Sud du delta du Saloum au Rio Geba. Teledetection, vol. 8, n² 2, p. 93-118.

Ariori, S. L., Ozer P., 2005. Évolution des ressources forestières en Afrique de l'Ouest soudanosahélienne au cours des 50 dernières années. Geo-Eco-Trop, vol. 29, p. 61-68.

Bassene O A., Cubizolle H., Cormier-Salem M-C., Sy B. A., 2013. L'impact des changements démographiques et socio-économiques sur la perception et la gestion de la mangrove en Basse Casamance (Sénégal). Géocarrefour, vol. 88, n 4, p. 299-315.

Bassène O., 2016. L'évolution des mangroves de la Basse Casamance au sud du Sénégal au cours des 60 dernières années : surexploitation des ressources, pression urbaine et tentatives de mise en place d'une gestion durable. Thèse de l'Université de Lyon-Saint Etienne, $310 \mathrm{p}$

Bindu K. K, 2019. Exercice de la souveraineté permanente d'un État sur ses ressources naturelles. Quelle gestion des ressources naturelles transfrontalières à l'est de la République Democratique Du Congo ? Meritum, Revista de Direito da Universidade FUMEC, vol. 14, $\mathrm{n}^{\circ}$ 1. P. 18-37

Binzangi K., Tshibangu K., Degreef J., Malaisse F., 1994. Le déboisement en Afrique tropicale. DéfisSud, p. 36-37.

Cohen W. B., Fiorella M., 1998. Comparison of methods for detecting conifer forest change with Thematic Mapper imagery. Remote sensing change detection: Environmental monitoring methods and applications, p. 89-102.

Coppin P., Jonckheere I., Nackaerts K., Muys B., Lambin E., 2004. Change detection methods in ecosystem monitoring: A review, International Journal of Remote Sensing [En ligne], vol. 25, $\mathrm{n}^{\circ} 9$, p. 1565-1596. DOI: https://doi.org/10.1080/0143116031000101675

Cormier-Salem M-C., 1999. Des littoraux aux limites mouvantes. In Cormier Salem M. C. (dir), Rivières du Sud. Sociétés et mangroves ouest-africaines. IRD Éditions, p. 17-30.

Deng J. S., Wang K., Deng Y. H., Qi G J., 2008. PCA-based land - use change detection and analysis using multitemporal and multisensor satellite data. International Journal of Remote Sensing [En ligne], vol. 29, $\mathrm{n}^{\circ}$ 16, p. 4823-4838. DOI: https://doi.org/10.1080/01431160801950162

Devineau J.-L., 1990. Propriétés spectrales de la végétation. In Pouget M., Mering C., Andrianasolo H. (ed.), Images satellite et milieux terrestres en régions arides et tropicales. Ed. ORSTOM, p. 43-49.

Diday E., 1971. Une nouvelle méthode de classification automatique et reconnaissance des formes : la méthode des nuées dynamiques. Revue de statistique appliquée, vol. 19, n² 2, p. 19-33.

Diedhiou I., 2019. Entre utilisation et préservation des ressources ligneuses en Afrique de l'Ouest: dynamiques des paysages forestiers en Sénégambie méridionale. Universités de Paris et Assane Seck de Ziguinchor, $444 \mathrm{p}$. 
Diedhiou I., Mering C., 2019. D'une rive à l'autre du fleuve : changements d'occupation du sol et dires d'acteurs en Basse Casamance. L'Espace géographique, vol. 48, n² 2, p. 117-137

Ebere C., 2015. La géopolitique du conflit en Casamance : les relations entre le Sénégal et la Gambie. In Conflit et paix en Casamance : dynamiques locales et transfrontalières, Gorée Institute, p. 56-74.

Fayama T., Dabiré D., Ba A., 2020. Perceptions du changement climatique et stratégies paysannes d'adaptation (zones soudaniennes du Burkina Faso et du Mali). In Sultan B., Bossa A. Y., Salack S., Sanon M. (dir.), Risques climatiques et agriculture en Afrique de l'Ouest. IRD Éditions, p. 289-296

Fichera C. R., Modica G., Pollino M., 2012. Land Cover classification and change-detection analysis using multi-temporal remote sensed imagery and landscape metrics. European Journal of Remote Sensing, [En ligne] vol. 45, n 1, p. 1-18. DOI: https://doi.org/10.5721/EuJRS20124501

Franklin S. E., Lavigne M. B., Wulder M. A., Stenhouse G. B., 2002. Change detection and landscape structure mapping using remote sensing. The Forestry Chronicle, vol. 78, $\mathrm{n}^{\circ}$ 5, p. 618-625

Giraut F., Guyot S., Houssay-Holzschuch M., 2004. Les aires protégées dans les recompositions territoriales africaines. L'Information géographique, $\mathrm{n}^{\circ} 4$, p. 340-368

Griffith J. A., Stehman S. V., Sohl T. L., Loveland T. R., 2003. Detecting trends in landscape pattern metrics over a 20-year period using a sampling-based monitoring program, International Journal of Remote Sensing, [En ligne] vol. 24, n 1, p. 175-181. DOI: https://doi.org/10.1080/01431160305009

Hayes D. J., Sader, S. A., 2001. Comparison of change-detection techniques for monitoring tropical forest clearing and vegetation regrowth in a time series. Photogrammetric engineering and remote sensing, vol. 6, nº 9, p. 1067-1075.

Hernandez I. E. R., Shi W., 2018. A Random Forests classification method for urban land-use mapping integrating spatial metrics and texture analysis. International Journal of Remote Sensing, [En ligne] vol. 39, n 4, p. 1175-1198. DOI: https://doi.org/10.3390/rs12172817

Joly G., 1987. Traitement informatique de l'image satellitaire. Bulletin de l'EPI (Enseignement Public et Informatique), vol. 47, p. 233-238.

Kpedenou D. K., Drabo O., Ouoba P. A., Da C. E. D., Tchamie T. T. K., 2017. Analyse de l'occupation du sol pour le suivi de l'évolution du paysage du territoire Ouatchi au sud-est Togo entre 1958 et 2015. Cahiers du Cerleshs, Presses de l'Université de Ouagadougou, vol. 31, nº 55, p. 203-228.

Lawrence P.-J., Chase T.-N., 2010. Investigating the climate impacts of global land cover change in the community climate system model. International Journal of Climatology, vol. $30, \mathrm{n}^{\circ} 13$, p. 2066-2087.

Le Hégarat-Mascle S., 2003. Classification methods of agronomic cultures (microwave and visible / infrared electromagnetic wave lengths) Nature Sciences Sociétés. vol. 11, n 1, p. 83-86.

Loyer J-Y., Boivin P., Le Brusq J.-Y., Zante P., 1988. Les sols du domaine fluvio-marin de Casamance (Sénégal). Évolution récente et réévaluation des contraintes majeures pour leur mise en valeur. In Selected Papers of the Dakar Symposium on Acid Sulphate Soils, $n^{\circ} 44$, p. 16-23.

Lu D., Mausel P., Brondízio E., Moran E., 2004. Change detection techniques. International Journal of Remote Sensing [En ligne], vol. 25, n 12, p. 2365-2401. DOI: https://doi.org/ $10.1080 / 0143116031000139863$

Lupo F., Reginster, I., Lambin E. F., 2001. Monitoring land-cover changes in West Africa with SPOT Vegetation: impact of natural disasters in 1998-1999. International Journal of Remote Sensing, vol. 22, $\mathrm{n}^{\circ} 13$, p. 2633-2639. 
Marut J. C., 2013. Wade et la Casamance. Un échec paradoxal. In Momar-Coumba Diop (dir.) le Sénégal sous Wade : le Sopi à l'épreuve du pouvoir. CRES-Karthala, Tome 2, p. 215-248.

Marut J-C., 2011. À l'Ouest, quoi de nouveau ? Les obstacles à la paix en Casamance. Les Cahiers d'Outre-Mer, n² 255, p. 363-376.

Demaze M. T., 2007. Le monitoring de la forêt amazonienne par télédétection. Hal archives-ouvertes [En ligne] : https://halshs.archives-ouvertes.fr/halshs-00315775

Montagne P., Oumarou I., Bertrand A., Rives F., Ichaou A., Peltier R., 2016. Bois-énergie domestique, démographie et urbanisation : situation après vingt-cinq années de gestion forestière des néo-communs au Sud-Niger. AFD. Conférence internationale de l'AFD sur le développement - $12^{\text {ème }}$ Edition, p. 1-14.

Ndiaye S., Charahabil M. M., Diatta M., 2017. Caractérisation des plantations à base d'anacardier (anacardium occidentale 1.) Dans le Balantacounda : cas des communes de Kaour, Goudomp et Djibanar (Casamance/Sénégal). European Scientific Journal Edition, vol. 13, n 12, p. 242-257

Oszwald J., Lefebvre A., Arnauld de Sartre X., Thales M. et Gond V., 2010. Analyse des directions de changement des états de surface végétaux pour renseigner la dynamique du front pionnier de Maçaranduba (Pará, Brésil) entre 1997 et 2006. Télédétection, vol. 9, n² 2, p. 97-111.

Ozer, P., 2004. Bois de feu et déboisement au Sahel : mise au point. Sécheresse, vol. 15, n 3, p. 243-251.

Peltier R., 2019. Quelles évolutions pour la consommation d'énergie domestique et la gestion des ressources naturelles dans les bassins d'approvisionnement des grandes villes du Sud ? Bois et Forêts des Tropiques [En ligne], vol. 340, p. 3-12. DOI: https://doi.org/10.19182/bft2019.340.a31708

Petit C.C., Lambin E.F., 2001. Integration of multi-source remote sensing data for land cover change detection. International Journal of Geographical Information Science, [En ligne] vol. 15, $\mathrm{n}^{\circ} 8$, p. 785-803. DOI: https://doi.org/10.1080/13658810110074483

Robin N., 2006. Le déracinement des populations en Casamance. Revue européenne des migrations internationales, Dossier : Figures et expériences diasporiques, vol. 22, nº 1, p. 153-181.

Samb C. O., Touré M. A., Faye El. H., Ba H. S., Diallo A. M., Badiane S., Sanogo D., 2018. Caractéristiques sociodémographique, structurale et agronomique des plantations d'anacardier (Anacardium occidentale L.) du Bassin arachidier et de la Casamance / Sénégal. Journal of Animal \& Plant Sciences, vol. 38, $\mathrm{n}^{\circ}$ 3, p. 6307-6325.

Sané T., 2017. Vulnérabilité et adaptabilité des systèmes agraires à la variabilité climatique et aux changements sociaux en Basse-Casamance (Sud-Ouest du Sénégal). Université Paris Diderot (Paris) Université Cheikh Anta Diop (Dakar), 358 p.

Sène A. M., 2019. Mobilités, activités socio-économiques et trafics à la frontière sénégalogambienne dans le contexte du conflit casamançais, Les analyses de Population \& Avenir, vol. 17, $\mathrm{n}^{\circ} 13$, p. 1-16.

Si Salah H., Goldin S. E., Rezgui A., Nour El Islam B., Ait-Aoudia S., 2020. What is a remote sensing change detection technique? Towards a conceptual framework. International Journal of Remote Sensing [En ligne], vol. 41, n 5, p. 1788-1812. DOI: https://doi.org/10.1080/01431161.2019.1674463

Sikuzani Y., Malaisse U., Kaleba F., Cabala S., 2017. Le rayon de déforestation autour de la ville de Lubumbashi (Haut-Katanga, RD Congo) : synthèse. Tropicultura, vol. 35, n 3, p. 215-221.

Solly B., Dieye E.H.B., Sy O., 2020. Remote Sensing Mapping of Land Use and Land Cover Changes in Upper Casamance, Senegal, 1987-2018. American Journal of Remote Sensing, vol. 8, n 2, p. 35-49 
Solly B., Dieye EL Hadji B., Sy O., 2018. Suivi de la déforestation par télédétection Hauterésolution dans le département de Médina Yoro Foulah (Haute-Casamance, Sénégal). Journal International en Sciences et Techniques de l'Eau et de l'Environnement, vol. 3, n 2, p. 38-41.

Soumare S., Fall, A., Andrieu J., Marega, O., Dieme B., 2020. Dynamique spatio-temporelle de la mangrove de Kafountine dans l'estuaire de la Basse-Casamance des années 1972 à nos jours. Approche par télédétection. IOSR Journal of Engineering, vol. 10, p. 1-14.

Tang Y., Atkinson P-M., Wardrop N-A., Zhang J., 2013. Multiple-point geostatistical simulation for post-processing a remotely sensed land cover classification Spatial Statistics, vol. 5, p. 69-84

Tavares E., 2015. La géopolitique du conflit en Casamance : relations avec les États voisins de Gambie et de Guinée-Bissau. In Conflit et paix en Casamance : dynamiques locales et transfrontalières, Gorée Institute, p. 43-45.

Tendeng M., Ndour N., Sambou B., Diatta M., Aouta, A., 2016. Dynamique de la mangrove du marigot de Bignona autour du barrage d'Affiniam (Casamance, Sénégal). International Journal of Biological and Chemical Sciences, vol. 10, $\mathrm{n}^{\circ} 2$, p. 666-680.

Treanor N. B., 2015. China's Hongmu Consumption Boom: Analysis of the Chinese Rosewood Trade and Links to Illegal Activity. In Tropical Forested Countries in Forest Trends Report Series, Forest Trade and Finance. $\mathrm{P} 48$.

Zitova B., Flusser J., 2003. Image registration methods: a survey. Image and Vision Computing, vol. $21, \mathrm{n}^{\circ} 11$, p. $977-1000$.

\section{NOTES}

1. Depuis 1982, cette région fait l'objet d'une revendication indépendantiste formulée par le Mouvement des forces démocratiques de Casamance (MFDC).

2. Constitué entres autres de Detarium senegalense, Daniellia oliveri, Bombax costatum, Stercularia setigera, Pterocarpus erinaceus, Parkia biglobosa, Dalium guineense, Terminalia macroptera, Khaya senegalensis Cola cordifolia et Adansonia digitata.

3. Chenal d'eau ou petit affluent salé et généralement bordé de mangroves, caractéristique des estuaires du Sénégal et de la Gambie.

4. Les tannes désignent les surfaces, nues ou couvertes d'halophytes de petite taille, formées naturellement aux dépens de la mangrove. On les trouve soit dans la zone située entre la mangrove et la terre ferme, de manière continue ou discontinue, soit au sein même des peuplements de palétuviers.)

5. La résolution des images MSS est d'environ 80 mètres alors que les images TM, ETM+ et OLI ont une résolution de 30 mètres.

6. À titre d'exemple, la scène du 26 novembre 2016 est plus grande que celle du 8 décembre 2000 avec respectivement 7771 colonnes contre 6999.

7. En effet, cette première étape de classification par type de milieu bénéficie des avantages de la méthode adoptée dans laquelle la séparation des classes repose sur les contrastes entre les réponses radiométriques d'un même ensemble de pixels déterminé par l'opérateur. Ici, la classification ne s'appliquera pas à la totalité des pixels de l'image mais à un sous-ensemble (pixels de milieu humide ou pixels de terre ferme)

8. Les tannes vifs sont totalement dépourvus de végétation ce qui explique l'absence de pic dans la proche infrarouge; ils sont parfois recouverts d'une croute de sel ce qui explique leur teinte très claire et donc leur forte luminance dans le visible et ont une très faible teneur en eau, d'où une forte réponse dans le moyen infrarouge. 
9. Les logiciels de Télédétection (ici Idrissi) limitent en général à 15 ou 16 le nombre de classes demandé par l'opérateur en sortie de la classification par K-moyennes.

10. Un tel choix nous prive de la possibilité d'analyser l'évolution du trait de côte mais tel n'a pas été ici notre objectif

11. Précisons que le terme « rizières de plateau » fait référence ici aux rizières douces de fin de pente, peu profondes et généralement soumises à l'influence d'une nappe phréatique d'eau douce (Loyer et al., 1988).

12. Voir tableau 2.

13. Voir tableau 2.

14. A titre d'exemple le prix incitatif du kilogramme de noix de cajou est passé de 25 FCFA en 1987 à 185 FCFA en 1998, puis de 400 FCFA en 2007 à 900 FCFA en 2017.

15. Cette partie frontalière avec la Guinée Bissau a été pendant longtemps le théâtre de combats intenses entre le MFDC et l'armée sénégalaise ou bissau-guinéenne avec comme conséquence majeure l'usage massif de mines. L'intensité des affrontements entre groupes armés a conduit à la désertion de ces zones par les populations qui se sont réfugiées en Guinée-Bissau ou dans les centres urbains comme Ziguinchor. Suite à l'accalmie de ces dernières années, des retours de réfugiés sont observés dans certaines zones, mais force est de constater aussi que la plupart des zones sont aujourd'hui inaccessibles en raison de la présence d'engins explosifs.

\section{RÉSUMÉS}

Cet article présente une méthode de cartographie par Télédétection de l'occupation du sol et de ses changements à partir du cas de la Sénégambie méridionale. L'approche rétrospective adoptée s'appuie sur la comparaison entre les signatures spectrales dites de «référence " issues des vérités de terrain et celles des classes résultant de la classification multispectrale non supervisée des images prises à des dates antérieures. Le résultat est une cartographie des principales catégories d'occupation du sol à chacune des dates étudiées et de leur évolution dans le temps. Ont ainsi été mis en évidence les principaux changements des paysages forestiers sénégambiens : un important recul des boisements de terre ferme au nord de la région au cours de la dernière décennie et une régénération de la mangrove suite à l'amélioration des conditions pluviométriques.

This article presents a method of mapping by remote sensing of land cover and its changes based on the case of Southern Senegambia. The retrospective approach adopted is based on the comparison between the so-called "reference" spectral signatures resulting from ground truths and those of the classes resulting from the unsupervised multispectral classification of images taken at earlier dates. The result is a cartography of the main land cover categories at each of the dates under study and their evolution over time. The main changes in the Senegalese forest landscape were thus highlighted: a significant decline in land afforestation in the north of the region over the last decade and a regeneration of the mangrove following the improvement in rainfall conditions. 


\section{INDEX}

Mots-clés : cartographie des changements, Image Landsat, classification multispectrale, occupation du sol, Sénégambie méridionale

Keywords : Land cover change mapping, Landsat image, multispectral classification, land use, Southern Senegambia

Thèmes : Sur le Champ - Sur le Terrain

\section{AUTEURS}

\section{IBRAHIMA DIÉDHIOU}

Ibrahima Diédhiou, ibrahima.diedhiou85@gmail.com, est attaché temporaire d'enseignement et de recherche à l'Université de Lorraine, Laboratoire LOTERR. Il a récemment publié :

- San Emeterio J L., Marega O., Sane T., Fall., Diédhiou I., Andrieu J., 2018. La Cartographie de l'environnement sahélien, des préjugés scientifiques aux choix de méthodes cartographiques. In La face cachée des cartes, Actes Colloque, p. 273-285

- Sané T., Méring C., Cormier-Salem M-C., Diédhiou I., Ba B-D., Diaw A-T., Tine AK., 2018.

Permanences et mutations dans les terroirs rizicoles de Basse-Casamance (SÉNÉGAL). L'Espace géographique, vol. 47, p. 201-218.

- Diédhiou I., Méring C., 2019. D'une rive à l'autre du fleuve : Changements d'occupation du sol et dires d'acteurs en Basse-Casamance, L'Espace géographique, vol. 48, p. 117-137.

\section{CATHERINE MERING}

Catherine Mering, cmering.up7@gmail.com, est Professeure émérite à l'Université de Paris. Elle a récemment publié :

- Bidou J.E., Droy I., Houéssé R., Mering C., 2018. Dynamiques démographiques, vulnérabilité et évolution du couvert végétal au nord Bénin : des interactions complexes. Espaces, Populations, Sociétés, 3-4.

- Diédhiou I., Mering C., 2019. D'une rive à l'autre du fleuve : changements d'occupation du sol et dires d'acteurs en Basse Casamance. L'Espace géographique, vol. 48, p. 117-137.

- Alexandre F., Mering C, 2019. Perception et représentation des changements socioenvironnementaux dans les sociétés rurales en Afrique de l'Ouest sahélienne et soudanienne.

L'Espace géographique, vol. 48, p. 97-102.

\section{OUMAR SY}

Oumar SY, oumarsy@univ-zig.sn, est Professeur à l'Université Assane Seck (Ziguinchor). Il a récemment publié :

- Mballo I., Sy O., 2020. Dynamiques spatio-temporelles des villes secondaires au Sénégal : les cas de Médina Gounass et de Diaobé en Haute Casamance de 1965 à 2018. African Journal on Land Policy and Geospatial Sciences, vol. $3 \mathrm{n}^{\circ}$ 2, p. 110- 122.

- Solly B., Dieye El. H, Mballo I, Sy O., Sane T., Thior M., 2020. Dynamique spatio-temporelle des paysages forestiers dans le Sud du Sénégal : cas du département de Vélingara. Physio-Géo [En ligne], Vol. 15. URL: http://journals.openedition.org/physio-geo/10634 - DOI: https://doi.org/ $10.4000 /$ physio-geo.10634

- Solly B., Dieye El. H, Sy O., 2020. Remote Sensing Mapping of Land Use and Land Cover Changes in Upper Casamance, Senegal, 1987-2018. American Journal of Remote Sensing. vol. 8, n 2, p. 35-49. 


\section{TIDIANE SANÉ}

Tidiane Sané, tsane@univ-zig.sn, est habilité à diriger des recherches à l'Université Assane Seck de Ziguinchor. Il a récemment publié :

- Sané T., Méring C., Cormier-Salem M-C., Diédhiou I., Ba B-D., Diaw A-T., Tine AK., 2018).

Permanences et mutations dans les terroirs rizicoles de Basse-Casamance (SÉNÉGAL). L'Espace géographique, vol. 47, $\mathrm{n}^{\circ}$ 3, p. 201-218.

- Badiane A., Sané T., Thior M., 2019. Impacts de la Dynamique des Paysages Agraires sur les Activités Agricoles dans la Commune d'adéane en Basse-Casamance (Sénégal). European Scientific Journal [En ligne], vol. 15. n² 21, p 489-506. DOI: https://doi.org/10.19044/esj.2019.v15n21p489 - Thior M., Sané T., Dièye, El H., Sy O., Cissokho D., Ba B D., Descroix L., 2019. Coastline dynamics of the northern Lower Casamance (Senegal) and southern Gambia littoral from 1968 to 2017. Journal of African Earth Sciences [En ligne], n 160. DOI: https://doi.org/10.1016/j.jafrearsci. 2019.103611 